\title{
Highly-functionalised difluorinated (hydroxymethyl)conduritol analogues via the Diels-Alder reactions of a difluorinated dienophile
}

\author{
Andrea Arany, ${ }^{a}$ Patrick J. Crowley, ${ }^{b}$ John Fawcett,${ }^{c}$ Michael B. Hursthouse, ${ }^{d}$ \\ Benson M. Kariuki, ${ }^{a}$ Mark E. Light, ${ }^{d}$ Andrew C. Moralee, ${ }^{c}$ Jonathan M. Percy ${ }^{* c}$ and \\ Vittoria Salafia ${ }^{c}$ \\ ${ }^{a}$ School of Chemical Sciences, University of Birmingham, Edgbaston, Birmingham, \\ UK B15 2 TT \\ ${ }^{b}$ Syngenta Ltd., Jealott's Hill International Research Centre, Bracknell, Berks, UK RG42 $6 E Y$ \\ ${ }^{c}$ Department of Chemistry, University of Leicester, University Road, Leicester, UK LE1 7 RH. \\ E-mail: jmp29@le.ac.uk; Fax: +44116252 3789; Tel: +44 1162522140 \\ ${ }^{d}$ EPSRC National X-Ray Crystallography Service, Department of Chemistry, \\ University of Southampton, Highfield, Southampton, UK SO17 1 BJ
}

Received 10th November 2003, Accepted 3rd December 2003

First published as an Advance Article on the web 21st January 2004

A difluorodienophile, synthesised using a Stille coupling reaction underwent tin(IV)-catalysed cycloaddition with three furans to afford oxa[2.2.1]bicycloheptenes in good yield. Reduction of ester and carbamate carbonyl groups and diol protection as the acetonide set the stage for palladium-catalysed hydrostannylation in two cases. Treatment of the stannanes with methyllithium triggered ring-opening to afford highly-functionalised difluorinated cyclohexenols which could be deprotected to afford (hydroxymethyl)conduritol analogues.

\section{Introduction}

Fluorination of cyclitols is a well established tactic for modifying functional group behaviour, disrupting or identifying essential hydrogen bonding interactions and preventing phosphorylation or other conjugation at key sites. ${ }^{1}$ There are two strategies available for the synthesis of fluorinated carbohydrates or cyclitols. Fluorinations with DAST or DeoxoFluor, ${ }^{2}$ which transform hydroxyl or ketonic carbonyl groups with the incorporation of one or two fluorine atoms, are very well established. There are many successful examples in the literature, particularly from readily-available natural product starting materials. ${ }^{3}$ However, this transformative approach does present a number of problems. Though there are some spectacular examples of selective fluorinations of unprotected substrates with DAST, ${ }^{4}$ extensive functional or protecting group manipulations are usually required to present a single group to the reagent, and even then, the course of fluorination reactions can be unpredictable. Fluorinations of carbonyl groups with DAST, involve transition states in which electron demand is relatively high, leading to the activation of pathways such as neighbouring group participation, group shifts and elimination reactions. ${ }^{5}$ Complex mixtures of products can result, though some imaginative solutions have been developed to minimise competing pathways. ${ }^{6}$

De novo methods using building blocks for the construction of difluorinated analogues of monosaccharides, carbasugars or cyclitols are not well established. There are successful strategies that deliver fluorinated 6-membered carbocycles based on free radical cyclisations ${ }^{7}$ and intramolecular aldol reactions, ${ }^{8}$ but the most obvious disconnection of highly-functionalised cyclohexane derivatives would rely on the availability of reliable Diels-Alder cycloaddition chemistry ${ }^{9}$ based on either difluorinated dienes, ${ }^{10}$ or difluorinated dienophiles. ${ }^{11}$ For the construction of cyclitol analogues, dienes such as alkoxybutadienes or furans are the key components, rather than the much more reactive cyclopentadiene.

The perfluoro effect ${ }^{12}$ indicates that FMO energies should be perturbed minimally by the presence of one ${ }^{13}$ or two fluorine atom alkene substitutents, though the experimental evidence arising from solution reactivity is extremely limited. If the statement of the effect is correct, and if Diels-Alder reactivity is controlled by the size of the dienophile LUMO/ diene HOMO gap, ${ }^{14}$ no special reactivity should accrue to difluorinated dienophiles, though they should at least compete effectively with their non-fluorinated analogues. On the other hand, significant differences may arise between alkenes and difluoroalkenes if alkene bond energies are most important in determining the activation energies and equilibrium constants of furan Diels-Alder reactions. However, difluorinated dienophiles are also potent Michael acceptors ${ }^{15}$ leading to relatively facile hydrolysis and other adventitious reactions with nucleophiles, imposing a potential restriction on the scope of available Diels-Alder chemistry. This high reactivity may be a function of the presence of fluorine atom leaving groups $\beta$-to a $\pi$-acceptor, rather than reflecting any intrinsic high electrophilicity due to a low-lying LUMO.

Wakselman et al ${ }^{16}$ made the most significant contribution in the area by synthesising ethyl 3,3-difluoroacrylate 1 from dibromodifluoromethane and ethyl vinyl ether (Scheme 1). The synthesis (based on an original procedure by Tarrant) was concise and scaleable, and a reaction with furan delivered cycloadducts 2 in $40 \%$ yield (endo : exo $4: 1$ ).

The reaction requires a relatively high loading of zinc iodide as Lewis acid ${ }^{17}(43 \mathrm{~mol} \%)$ and an excess of furan (5.65 equiv-

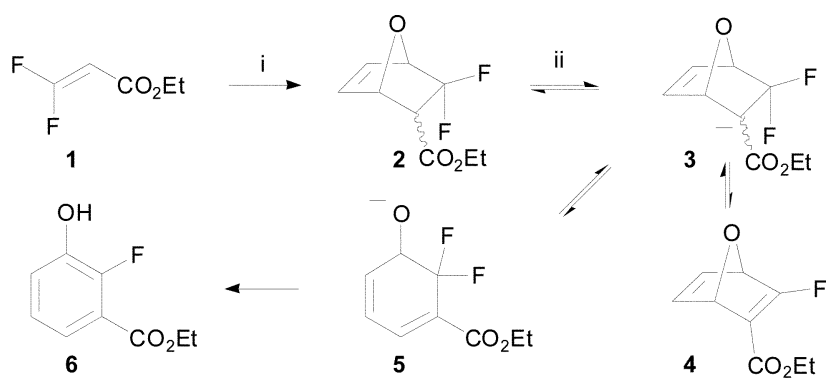

Scheme 1 i) $\mathrm{ZnI}_{2}$, furan, hydroquinone, $80{ }^{\circ} \mathrm{C}, 85 \mathrm{~h}$; (ii) $\mathrm{TBAF} \cdot 3 \mathrm{H}_{2} \mathrm{O}$, $45^{\circ} \mathrm{C}, 2 \mathrm{~h}$. 
alents), and starting material was consumed slowly (over 3 days). In the absence of catalyst, the fluorinated substrate was an order of magnitude more reactive than ethyl acrylate, which took one month to reach $20 \%$ conversion, which is interesting given the anticipated minimal FMO perturbation. Ring opening was attempted directly from 2 via the conjugate base but only phenol $\mathbf{6}$ could be isolated as the major product. The use of fluoride as base allows the repopulation (from 4) of carbanion $\mathbf{3}$ from which ring opening to $\mathbf{5}$ can proceed with strain relief; subsequent loss of HF results in aromatisation.

A number of issues are addressed in this manuscript; firstly, we wished to synthesise and explore the reactivity of a difluorinated dienophile 8 from which cycloadduct dehydrofluorination was not possible. Inexpensive trifluoroethanol was chosen as our starting material and we wished to react the dienophile ${ }^{18}$ with representative substituted furans, and compare its reactivity qualitatively with that of the non-fluorinated analogue. Most importantly, we wished to show that cycloadduct ringopening was achievable without aromatisation. Furans were selected as dienes for the study because of their low nucleophilicity in stepwise polar reactions (compared to other heteroatom-substituted dienes ${ }^{19}$ ) and also because their cycloadducts have a rich chemistry. ${ }^{20}$

\section{Results and discussion}

Alkenoate 8 was synthesised from stannane 7 (which is prepared from trifluoroethanol ${ }^{21}$ ) and a large excess of ethyl chloroformate under modified Farina-Liebeskind conditions (Scheme 2). ${ }^{22}$ Couplings were carried out on up to 0.1 mole of stannane, delivering alkenoate in good reproducible yield (70 $80 \%){ }^{23}$ Purification was achieved by filtration through a short plug of silica followed by Kugelrohr distillation. All attempts to purify or improve the quality of the chloroformate led to the formation of significant quantities of enol carbamate 9 from coupling reactions. Slow addition of the chloroformate was also essential; 9 formed a major part of the product when the chloroformate was added rapidly or in one portion. Dimer $\mathbf{1 1}$ could also be identified as a minor by-product in some coupling reactions. Oxidative coupling either of 7 , or of the corresponding vinylcopper species (presumably the active carrier in the transmetallation from tin to palladium) would account for the formation of this species.

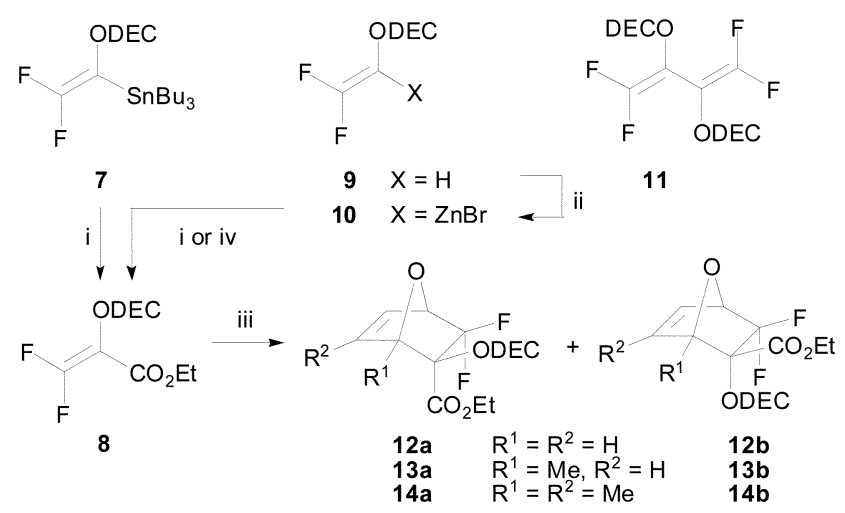

Scheme 2 (i) $\mathrm{ClCO}_{2} \mathrm{Et}, \mathrm{Pd}(\mathrm{OAc})_{2}, \mathrm{CuI}, \mathrm{Ph}_{3} \mathrm{P}, \mathrm{THF}, 60^{\circ} \mathrm{C}, 2 \mathrm{~h}$ (ii) $t$ - $\mathrm{BuLi}, \mathrm{THF},-78{ }^{\circ} \mathrm{C}$ then $\mathrm{ZnCl}_{2}$, warm to rt; (iii) furan, 2methylfuran or 2,3-dimethylfuran, $\mathrm{SnCl}_{4}, \mathrm{CH}_{2} \mathrm{Cl}_{2}$, rt or $0{ }^{\circ} \mathrm{C}$; (iv) $\mathrm{ClCO}_{2} \mathrm{Et}, \mathrm{Pd}_{2} \mathrm{dba}_{3} \cdot \mathrm{CHCl}_{3}, \mathrm{Ph}_{3} \mathrm{P}, \mathrm{THF}, 50^{\circ} \mathrm{C}, 16 \mathrm{~h}$. DEC $=\mathrm{CONEt}_{2}$

The alkenoate could also be prepared from 9 via the vinylzinc reagent $10,{ }^{24}$ treatment of 9 sequentially with tert-butyllithium in THF at $-78^{\circ} \mathrm{C}$, then a solution of anhydrous zinc chloride in THF, followed by ethyl chloroformate under the catalytic conditions described above afforded $\mathbf{8}$ in acceptable yield. The coupling also proceeded successfully in the absence of the copper salt when $\mathrm{Pd}_{2} \mathrm{dba}_{3} \cdot \mathrm{CHCl}_{3}$ was used as the catalyst, though a longer reaction time was required. We have now adopted this procedure for the preparation of stocks of alkenoate in our laboratory; though the (unoptimised) yield was moderate $(52 \%)$, the alkenoate product can be distilled directly (Kugelrohr) without silica gel filtration and we avoid handling tin compounds in two reaction steps, and producing organotin liquid waste. Current work in our laboratory seeks further process improvements.

Catalyst screening procedures were described in our earlier communication $^{18 a}$ and will not be discussed fully here, but certain reactions require comment. Zinc iodide was a poor catalyst for the cycloaddition between $\mathbf{8}$ and furan, delivering a very low conversion to $\mathbf{1 2} \mathbf{a} / \mathbf{b}$ under the Wakselman conditions. Reactions with diethylaluminium chloride were more successful, delivering the highest endo : exo selectivity observed to date $(8: 1)$, but requiring a very large excess of furan (20 equivalents) and an extended reaction time. ${ }^{18 a}$ These conditions were used to prepare pure 12a for the first time which was crystallised for X-ray diffraction analysis allowing a correlation between the ${ }^{19} \mathrm{~F}$ NMR chemical shifts and the cycloadduct relative stereochemistry.

Preparative cycloadditions were carried out in the presence of stannic chloride ${ }^{25}(25 \mathrm{~mol} \%)$ in dichloromethane at room temperature or below, allowing the use of a much smaller excess of furan (2 equivalents). As we wished to develop chemistry in which substituted furans could be used as dienes, improvement of the reaction stoichiometry was of great importance. To date, the cycloaddition reaction has not been performed above the 60 mmol scale though the total yield of isolated purified products is reproducible between 70 and $80 \%$. The isolated yield of purified endo-cycloadduct is moderate and the modest endo preference (3.2: 1 based on total purified cycloadducts) is consistent with the behaviour of other $\alpha$-oxysubstituted dienophiles. ${ }^{26}$ Cyclic carbonate $\mathbf{1 5}$ (Fig. 1) was also isolated as a minor product $^{33}$ in the furan Diels-Alder reaction under stannic chloride catalysed conditions.

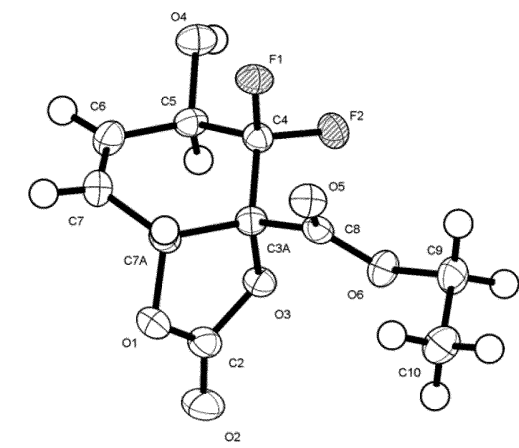

Fig. 1 ORTEP plot of cyclic carbonate $\mathbf{1 5}$ showing the atom label scheme and $50 \%$ displacement ellipsoids.

Presumably, the coordination of the Lewis acid to the bridging ether oxygen allows neighbouring group participation by the carbamoyloxy group; hydrolysis then delivers the cyclic carbonate 15 (Scheme 3).

The cycloaddition reaction rate was sensitive to solvent polarity in qualitative experiments; dichloromethane and 1,2dichloroethane appeared to be the best solvents with slower reactions occurring in chloroform or toluene. Cycloadducts were not detected following reactions in carbon tetrachloride, acetonitrile or trifluoroethanol. An attempt to carry out the cycloaddition in ionic liquid bmim $\cdot \mathrm{PF}_{6}$ led exclusively to decomposition of the alkenoate. Non-fluorinated analogue $\mathbf{1 6}$ (prepared from the corresponding stannane using chloroformate coupling) failed to deliver cycloadducts when reacted with furan under the conditions used for $\mathbf{8}$, and was returned from the reaction along with some polymerisation products.

A regioselective and more rapid cycloaddition reaction occurred with 2-methylfuran under the tin(IV)-catalysed conditions at $0{ }^{\circ} \mathrm{C}$, leading to the formation of a $1: 1$ (by ${ }^{1} \mathrm{H}$ NMR) 

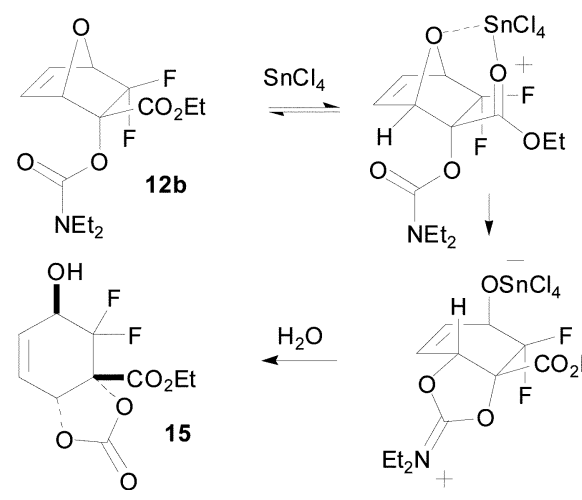

$\mathrm{H}_{2} \mathrm{O}$

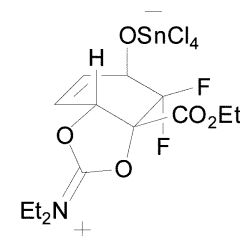

Scheme 3

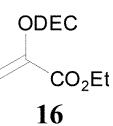

mixture of endo and exo cycloadducts in excellent isolated yield $(93 \%)$ with complete conversion of starting material. The reaction with 2,3-dimethylfuran was faster still; reacting 8 with a $1: 1$ mixture of 2-methyl and 2,3-dimethylfuran resulted in the exclusive formation of the more substituted cycloadduct with 2,3-dimethylfuran. The reaction was not stereoselective (1:1.4 by ${ }^{1} \mathrm{H}$ NMR) but the isolated yield was again excellent $(92 \%)$. We were unable to detect cyclic carbonate products analogous to $\mathbf{1 5}$ from the 2-substituted furans.

There are relatively few reports of Diels-Alder reactions of 2-methylfuran, though all concur upon its low reactivity, even under zinc iodide-catalysed conditions. Sneden reported ${ }^{27}$ that all simple dienophiles (except maleic anhydride) failed to undergo cycloaddition, whereas 2-methylfuran reacted with $\alpha$-acetoxyacrylonitrile successfully (though very slowly) in the hands of Vogel and Kernen. ${ }^{28}$ However, the reaction between 8 and this more electron-rich furan is faster than the reaction of 8 with furan itself.

The regiochemical outcome was assigned from the ${ }^{13} \mathrm{C}$ NMR spectrum initially, and confirmed by X-ray crystallographic analysis. Four distinct bridgehead carbons can be seen, and distinguished easily in the PENDANT experiment. The two tertiary bridgehead carbons (which bear methine protons) appear as triplets $\left({ }^{2} J_{\mathrm{C}-\mathrm{F}}=28 \mathrm{~Hz}\right)$ whereas the quaternary carbons are unsplit, consistent with the presence of regioisomers 13a and 13b; full analysis using HMBC experiments confirms the analysis. The regiochemistry of addition is entirely consistent with the observations of Vogel and Kernen who proposed a reaction mechanism with pronounced diradical character. Formally, alkenoates 8 and $\mathbf{1 6}$ are captodative dienophiles. ${ }^{29} \mathrm{~A}$ biradical mechanism, or transition state with considerable biradical character, would involve the collapse of $\mathbf{1 7}$ or $\mathbf{1 8}$; the observed products correspond to the collapse of $\mathbf{1 7}$. In the former case, an alkyl substitutent at the furan 2-position can stabilise the intermediate diradical character. However, in the latter case where $\mathrm{R}^{2}=\mathrm{Me}$, the alkyl substitutent is connected to the radical centre through the alkenyl group so the difference between the two possible biradical intermediates seems less clear cut. Our observations suggest that the effect of an alkyl group directly attached to the radical centre (the $\alpha$-allylic position) is much greater than one more remote (at the $\gamma$-position).
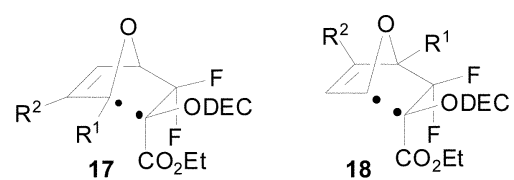

Cycloaddition reactions of 2,3-dimethylfuran are unusual; we found examples with maleic anhydride and DMAD only, ${ }^{30}$ while less reactive dienophiles such as acrolein undergo conju- gate addition. ${ }^{31}$ Because the yields for the cycloaddition reactions are good to excellent $(66-93 \%)$ and all reactions reach $100 \%$ conversion in $\mathbf{8}$, we propose that the role of the fluorine atoms could be to destabilise the alkene ground state, ${ }^{32}$ resulting in more facile progress to the transition state, and indeed, help to overcome the notorious reversibility of the furan DielsAlder reaction. Reversibility may exert a significant influence upon the failure of $\mathbf{1 6}$ to react if the equilibrium constant favours the aromatic furan and the alkene. These issues form the subject of current computational investigation and will be reported elsewhere.

In each 2-substituted furan case, there was signal broadening in the ${ }^{19} \mathrm{~F}$ NMR spectrum at ambient temperature for one stereoisomer, though the signals sharpened at $348 \mathrm{~K}$ in $\mathrm{d}^{6}$ benzene. The endo- and exo-cycloadducts from the reaction with the substituted furans were inseparable chromatographically but we were able to crystallise the exo-cycloadducts in both cases, determine the crystal structures and confirm that they gave rise to the broad signals in the ${ }^{19} \mathrm{~F}$ NMR spectra. Presumably the presence of the methyl group at the bridgehead position in cycloadducts $\mathbf{1 3 b}$ and $\mathbf{1 4 b}$ causes restricted rotation in the ester or carbamate groups.

Other heteroatom-containing dienes failed to undergo the cycloaddition reaction with $\mathbf{8}$. Danishefsky's diene, 1-trimethylsilyloxybutadiene, 1-acetoxybutadiene, 2-methoxy-, 2-acetoxyand 2-pivaloyloxy-furan all delivered complex mixtures of products under thermal, and Lewis acid catalysed conditions; in all cases, $[4+2]$ cycloadducts were very difficult to detect unequivocally in ${ }^{19} \mathrm{~F}$ NMR spectra of the crude products, and could not be isolated. These dienes are all considerably more nucleophilic than furan ${ }^{19}$ and may react through additionelimination pathways with $\mathbf{8}$.

Accurate and controlled ring-opening of oxa-[2.2.1]bicycloheptenes has been achieved by a variety of methods. We decided to apply the hydrostannylation methodology described by Lautens ${ }^{34}$ because it used only one equivalent of alkyllithium in the ring opening reaction and we were concerned about the potential lability of the allylic $\mathrm{CF}_{2}$ group in $\mathrm{S}_{\mathrm{N}} 2^{\prime}$ displacements. ${ }^{35}$ The cycloadducts themselves contain groups that are capable of reacting with the alkyllithiums used to trigger the stannate ring opening reactions so we decided to remove carbonyl groups from the substrates by reduction. Simultaneous ester and carbamate reduction from 12a was achieved with lithium aluminium hydride in THF at reflux, followed by protection of diols $\mathbf{1 9}$ as the acetonides $\mathbf{2 0}$ (Scheme 4). The inseparable endo- and exo-mixture $(\mathbf{1 3 a}, \mathbf{1 3 b})$ from the 2-methylfuran cycloaddition was reduced to the mixture of diols $(\mathbf{2 1 a}, \mathbf{2 1 b})$ which were separated as their acetonides (22a, 22b) (Table 1).

A crystal structure was obtained for 22a as proof of relative configuration and supporting the 2D NMR assignment of structure and cycloaddition regiochemistry.
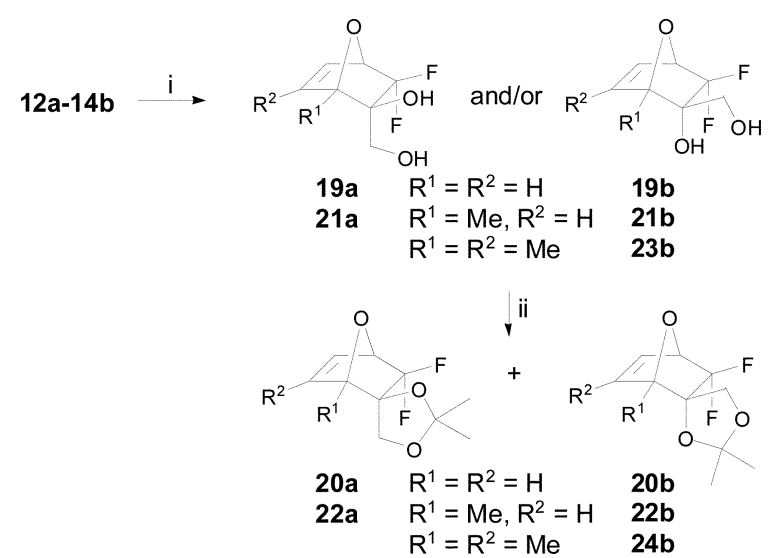

Scheme 4 i, $\mathrm{LiAlH}_{4}$, THF, reflux, $3 \mathrm{~h}$; ii acetone, $\mathrm{CuSO}_{4}, \mathrm{TsOH} \cdot \mathrm{H}_{2} \mathrm{O}$, $\mathrm{rt}, 12 \mathrm{~h}$. 
Table 1 Reduction of cycloadducts and protection of diols

\begin{tabular}{cccll}
\hline Adduct & Diol & $\%$ & Acetonide & $\%$ \\
\hline 12a & $\mathbf{1 9 a}$ & 68 & $\mathbf{2 0 a}$ & 77 \\
$\mathbf{1 3 a} / \mathbf{b}$ & $\mathbf{2 1 a / b}$ & 80 & $\mathbf{2 2 a}$ & 34 \\
& & & $\mathbf{2 2 b}^{a}$ & 37 \\
$\mathbf{1 4 b}$ & $\mathbf{2 3 b}$ & 89 & $\mathbf{2 4 b}$ & 91 \\
& & &
\end{tabular}

Table 2 Hydrostannylation, ring opening and deprotection reactions

\begin{tabular}{llrllll}
\hline Acetonide & Stannane & $\%$ & Alcohol & $\%$ & Triol & $\%$ \\
\hline 20a & $\mathbf{2 5}^{a}$ & 19 & $\mathbf{2 9}$ & 71 & $\mathbf{3 3}$ & 93 \\
& $\mathbf{2 6}$ & 38 & $\mathbf{3 0}$ & 40 & $\mathbf{3 4}$ & 95 \\
22a & $\mathbf{2 7}$ & 50 & $\mathbf{3 1}$ & 30 & $\mathbf{3 5}$ & 86 \\
22b & $\mathbf{2 8}$ & 56 & $\mathbf{3 2}$ & 65 & $\mathbf{3 6}$ & 92 \\
24b & - & 0 & & & &
\end{tabular}

${ }^{a}$ Separated at the stannane stage.

Hydrostannylations occurred smoothly for 20a, 22a and 22b under standard Lautens conditions (Scheme 5), ${ }^{34}$ provided tributyltin hydride was distilled freshly before use (Table 2).

The reaction was non-regioselective in the case of $\mathbf{2 0 a},{ }^{36}$ affording a $1: 1$ mixture (by integration of the ${ }^{19} \mathrm{~F}$ NMR spectrum) of $\mathbf{2 5}$ and $\mathbf{2 6}$, which could be separated by careful column chromatography.

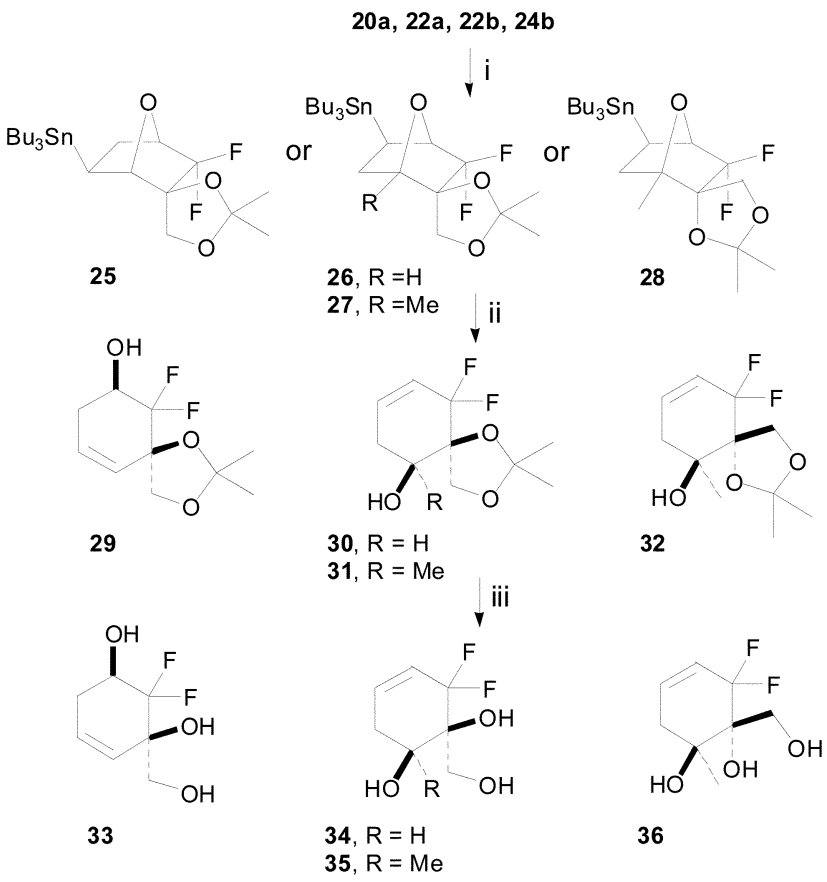

Scheme 5 (i) $\mathrm{Bu}_{3} \mathrm{SnH}, \mathrm{Pd}_{2} \mathrm{dba}_{3} . \mathrm{CHCl}_{3}, \mathrm{PPh}_{3}, \mathrm{PhMe}, \mathrm{rt}, 16 \mathrm{~h}$ (ii) $\mathrm{MeLi} \mathrm{OEt}_{2}, \mathrm{THF}, 0{ }^{\circ} \mathrm{C}, 20 \mathrm{~min}$; (iii) $\mathrm{MeOH}$, Amberlyst- $15,40^{\circ} \mathrm{C}$, $24 \mathrm{~h}$.

However, the recovery of $\mathbf{2 5}$ from chromatography was rather less efficient (19\% isolated yield). Both acetonides from 2-methylfuran afforded a single stannane consistent with the steric effect exerted by the methyl group at the bridgehead position. ${ }^{34}$ The acetonide $\mathbf{2 4 b}$ derived from $\mathbf{1 4 b}$ failed to undergo hydrostannylation; presumably steric hindrance prevents reaction in this case. The stannanes were very difficult to free completely from traces of other tributyltin compounds which were visible in the highfield region of the ${ }^{1} \mathrm{H}$ NMR spectra. Long range $\left({ }^{4} J_{\mathrm{H}-\mathrm{F}}\right)$ proton-fluorine couplings were another feature of the spectra of the stannanes. For example, in the ${ }^{1} \mathrm{H}$ NMR spectrum of 26, one of the methylene protons from the dioxolane ring appears as a doublet of triplets; the triplet splitting dis- appeared in the ${ }^{1} \mathrm{H}\left\{{ }^{19} \mathrm{~F}\right\}$ spectrum, consistent with its origin in coupling to both fluorine nuclei. The heterodecoupling experiment also allowed the separation of $J$ and ${ }^{3} J_{\mathrm{H}-\mathrm{Sn}}$ couplings in the bridgehead proton next to the $\mathrm{CF}_{2}$ centre, and simplification to a doublet, of the other bridgehead proton.

In preliminary ring opening reactions, one of the stannane regioisomers $\mathbf{2 6}$ had afforded a very low yield of ring opened product suggesting further destructive reaction of the ring opened product via formal $\mathrm{S}_{\mathrm{N}} 2^{\prime}$ loss of fluoride ion. When we exposed 25 and $\mathbf{2 6}$ to the Lautens conditions separately on a slightly larger scale $(1.5 \mathrm{mmol})$ following the reactions carefully by TLC, we found that both $\mathbf{2 9}$ and $\mathbf{3 0}$ could be obtained in better yields. The products gave good ${ }^{1} \mathrm{H},{ }^{13} \mathrm{C}$ and ${ }^{19} \mathrm{~F}$ NMR spectra directly after trituration with hexane. Full 2D NMR analysis was used to confirm the structure of 29; starting from the acetonide methyl groups, HMBC was used to show connectivity through into one of the alkene carbons, neither of which were split by fluorine. Structure $\mathbf{3 0}$ was assigned by the appearance of the alkene signals in the ${ }^{13} \mathrm{C}$ NMR spectrum; ${ }^{2} J_{\mathrm{C}-\mathrm{F}}(33 \mathrm{~Hz}, 24 \mathrm{~Hz})$ and ${ }^{3} J_{\mathrm{C}-\mathrm{F}}(13 \mathrm{~Hz}, 10$ $\mathrm{Hz}$ ) couplings were clearly observable. We believe that our earlier lower yield in the case of $\mathbf{3 0}$ arose from inadequate control of reaction stoichiometry or time on the smaller scale. Attempting to scale-up the direct reductive ring opening of 20a to 29 under the $\mathrm{MgBr}_{2} / t$ - BuLi reaction conditions $^{18 a}$ was considerably less successful with very complex mixtures of products obtained; significant yield losses occurred upon chromatography and we were unable to reproduce our modest $(30 \%)$ initial yield above the millimole scale. This direct ring-opening procedure was not pursued further. From the 2-methyl series, the crystal structure of $\mathbf{3 1}$ was solved and structure 32 was confirmed by full 2D NMR analysis. Deprotection of the acetonides 29-32 could be achieved under standard conditions to deliver the triols 33-36 which were straightforward to characterise by NMR methods. Apart from 33, all products of ring opening showed AB-type ${ }^{19} \mathrm{~F}$ NMR spectra with large $(200-250 \mathrm{~Hz}){ }^{2} J_{\mathrm{F}-\mathrm{F}}$ coupling constants. However, the ${ }^{19} \mathrm{~F}$ NMR spectrum of $\mathbf{3 4}$ appears as a superimposition of a singlet and a doublet (the splitting is a ${ }^{3} J_{\mathrm{H}-\mathrm{F}}$ ) indicating that the fluorine environments are magnetically very similar with ${ }^{2} J_{\mathrm{F}-\mathrm{F}}$ tending to zero. Complex signals were observed for alkene protons in most cases due to couplings to fluorine; for example the $400 \mathrm{MHz}{ }^{1} \mathrm{H}$ NMR spectrum of $\mathbf{3 4}$ shows multiplets for both alkene signals. However, the ${ }^{1} \mathrm{H}\left\{{ }^{19} \mathrm{~F}\right\}$ NMR spectrum revealed very clean doublets of triplets for both alkene signals, one showing a $3.8 \mathrm{~Hz}$ allylic coupling, and the other a $2.2 \mathrm{~Hz}$ homoallylic coupling, into the adjacent methylene group. The alkene proton assignment was confirmed by an HMQC experiment (again, one of the alkene carbon atoms shows a clear two bond coupling to fluorine, whereas the three bond coupling is not visible. The measurable alkene vicinal and the allylic and homoallylic coupling constants were used as the basis for spectral simulation experiments (with gNMR v 5.0). The alkenyl proton next to the $\mathrm{CF}_{2}$ centre appears as a dddt $\left(J 10.5,{ }^{3} J_{\mathrm{H}-\mathrm{F}} 7.7\right.$, $\left.3.10,{ }^{4} J 2.25,2.25\right)$ while its partner is a dtdd $(J 10.5,4.0,4.0$, $\left.{ }^{4} J_{\mathrm{H}-\mathrm{F}} 1.8,1.5\right)$. The heteronuclear decoupling experiment was also used to simplify the multiplet signal arising from the methine proton adjacent to the hydroxyl group, which shows four bond couplings to both protons. A triplet was observed in the ${ }^{1} \mathrm{H}\left\{{ }^{19} \mathrm{~F}\right\}$ NMR spectrum consistent arising from three bond couplings into the $\mathrm{OH}$, and one of the adjacent methylene pair.

\section{Conclusions}

A number of highly-substituted difluorinated cyclohexenols have evolved from the Diels-Alder reaction of $\mathbf{8}$ with furans. The reaction accepts alkyl substitution at C-2 and C-3 but acyclic heteroatom-substituted dienes failed to afford cyclo- 
adducts. These outcomes represent the first occasion on which a simple difluorinated building block has been used to construct highly hydroxylated cyclohexenes via cycloaddition chemistry. Alkenoate $\mathbf{8}$ is considerably more reactive towards furan than the non-fluorinated congener 16. The Lautens hydrostannylation/stannate ring opening strategy has provided an effective method for manipulating the cycloadducts through to richlyfunctionalised monocyclic species, though there are significant limits to the methodology. The presence of tin alkyl residues in advanced intermediates is a significant weakness of this method of ring-opening.

Current work seeks improvements in dienophile synthesis and more direct methods for ring-opening so that these unusual products can be available by routes and procedures which are more concise.

\section{Experimental}

\section{Crystallography $\dagger$}

\section{Crystal data for 12a}

$\mathrm{C}_{14} \mathrm{H}_{19} \mathrm{~F}_{2} \mathrm{NO}_{5}, M_{\mathrm{r}}=319.30$, monoclinic, space group $P 2_{1} / c$, lattice parameters: $a=16.3285(16), b=11.9385(11), c=$ 17.5557(13) А̊, $a=90.00, \beta=116.70(4), \gamma=90.00, Z=8, V=$ $3057.4(5) \AA^{3}, D_{\mathrm{c}}=1.387 \mathrm{~g} \mathrm{~cm}^{-3}, \mu(\mathrm{Mo} \mathrm{K} \alpha)=0.120 \mathrm{~mm}^{-1}$, $F(000)=1344, T=150(2) \mathrm{K}$, block, crystal dimensions $0.20 \times$ $0.20 \times 0.15 \mathrm{~mm}$.

\section{Crystal data for 13b}

$\mathrm{C}_{14} \mathrm{H}_{19} \mathrm{~F}_{2} \mathrm{NO}_{5}, M_{\mathrm{r}}=333.33$, triclinic, space group $P$-1, lattice parameters: $a=6.951(4), b=8.869(5), c=13.399(7) \AA, a=$ 98.346(10), $\beta=96.497(9), \gamma=91.913(10), Z=2, V=811.0(8) \AA^{3}$, $D_{\mathrm{c}}=1.365 \mathrm{~g} \mathrm{~cm}^{-3}, \mu($ Mo K $\alpha)=0.116 \mathrm{~mm}^{-1}, F(000)=352$, $T=150(2) \mathrm{K}$, block, crystal dimensions $0.14 \times 0.12 \times 0.06 \mathrm{~mm}$.

\section{Crystal data for $14 \mathrm{~b}$}

$\mathrm{C}_{16} \mathrm{H}_{23} \mathrm{~F}_{2} \mathrm{NO}_{5}, M_{\mathrm{r}}=347.35$, triclinic, space group $P$-1, lattice parameters: $a=7.8012(5), b=8.9989(5), c=14.1190(9) \AA, a=$ 96.881(3), $\beta=102.152(3), \gamma=108.900(3), Z=2, V=897.60(10)$ $\AA^{3}, D_{\mathrm{c}}=1.285 \mathrm{~g} \mathrm{~cm}^{-3}, \mu(\mathrm{Cu} \mathrm{K \alpha})=0.923 \mathrm{~mm}^{-1}, F(000)=368$, $T=296(2) \mathrm{K}$, plates, crystal dimensions $0.40 \times 0.30 \times 0.20 \mathrm{~mm}$.

\section{Crystal data for 15}

$\mathrm{C}_{10} \mathrm{H}_{10} \mathrm{~F}_{2} \mathrm{O}_{6}, M_{\mathrm{r}}=264.18$, triclinic, space group $P$-1, lattice parameters: $a=7.3657(7), b=7.5078(7), c=10.1384(9) \AA, a=$ 77.063(2), $\beta=78.383(2), \gamma=89.452(2), Z=2, V=534.86(9) \AA^{3}$, $D_{\mathrm{c}}=1.640 \mathrm{~g} \mathrm{~cm}^{-3}, \mu($ Mo K $\alpha)=0.157 \mathrm{~mm}^{-1}, F(000)=272$, $T=150(2) \mathrm{K}$, plates, crystal dimensions $0.35 \times 0.29 \times 0.08 \mathrm{~mm}$.

\section{Crystal data for 22a}

$\mathrm{C}_{11} \mathrm{H}_{14} \mathrm{~F}_{2} \mathrm{O}_{3}, M_{\mathrm{r}}=232.22$, monoclinic, space group $C c$, lattice parameters: $a=9.7681(13), b=11.8090(15), c=10.5162(15) \AA$, $\alpha=90.00, \beta=114.677(9), \gamma=90.00, Z=4, V=1102.3(3) \AA^{3}, D_{\mathrm{c}}=$ $1.399 \mathrm{~g} \mathrm{~cm}^{-3}, \mu($ Mo K $\alpha)=0.122 \mathrm{~mm}^{-1}, F(000)=488, T=200(2)$ $\mathrm{K}$, block, crystal dimensions $0.40 \times 0.40 \times 0.40 \mathrm{~mm}$.

\section{Crystal data for 31}

$\mathrm{C}_{11} \mathrm{H}_{16} \mathrm{~F}_{2} \mathrm{O}_{3}, M_{\mathrm{r}}=234.24$, monoclinic, space group $P 2_{1} / n$, lattice parameters: $a=9.8294(12), b=8.3435(13), c=13.9113(16) \AA$, $\alpha=90.00, \beta=98.998(10), \gamma=90.00, Z=4, V=1126.8(3) \AA^{3}, D_{\mathrm{c}}=$ $1.381 \mathrm{~g} \mathrm{~cm}^{-3}, \mu(\mathrm{MoK} \alpha)=0.120 \mathrm{~mm}^{-1}, F(000)=496, T=200(2)$ $\mathrm{K}$, block, crystal dimensions $0.40 \times 0.30 \times 0.30 \mathrm{~mm}$.

$\dagger$ CCDC reference numbers 224031-224036. See http://www.rsc.org/ suppdata/ob/b3/b314314g/ for crystallographic data in.cif or other electronic format.

\section{General}

NMR spectra were recorded on Bruker ARX-250, Bruker DPX-300, Bruker AC-300 or Bruker DRX-400 spectrometers. ${ }^{1} \mathrm{H}$ and ${ }^{13} \mathrm{C}$ NMR spectra were recorded using the deuterated solvent as the lock and the residual solvent as the internal reference. ${ }^{19} \mathrm{~F}$ NMR spectra were recorded relative to chlorotrifluoromethane as the external standard. The multiplicities of the spectroscopic data are presented in the following manner: app. = apparent, $\mathrm{s}=$ singlet, $\mathrm{d}=$ doublet, $\mathrm{t}=$ triplet, pent. $=$ pentet, $\mathrm{q}=$ quartet, $\mathrm{m}=$ multiplet and $\mathrm{br}=$ broad. The appearance of complex signals is indicated by $\mathrm{m}$. Homocouplings $(\mathrm{H}-\mathrm{H}, \mathrm{F}-\mathrm{F})$ are given in Hertz and specified by $J$; the nuclei involved in heteronuclear couplings are defined. Unless stated otherwise, all $J$ 's refer to ${ }^{3} J$ couplings. NMR spectral simulations were performed with gNMR v 5.0 (Adept Scientific).

Chemical ionisation (CI) mass spectra were recorded on Micromass Prospec or Kratos Concept 1H spectrometers using ammonia as the reagent gas. Electron impact (EI) spectra were recorded on Kratos MS-80, Micromass Prospec or Kratos Concept $1 \mathrm{H}$ spectrometers. Fast atom bombardment (FAB) spectra were recorded on a Kratos Concept $1 \mathrm{H}$ spectrometer at about $7 \mathrm{kV}$ using xenon and $m$-nitrobenzyl alcohol as the matrix. GC-MS was carried out on a Perkin Elmer TurboMass spectrometer fitted with a Zebron ZB-5 column $(30 \mathrm{~m} \times 0.25$ $\mu \mathrm{m})$ running a $20-350{ }^{\circ} \mathrm{C}$ ramp over 27 minutes. Electrospray (ES) mass spectra were recorded on Micromass LCT or Micromass Quattro LC spectrometers. High resolution mass spectrometry measurements were carried out either on the Micromass LCT or the Kratos Concept $1 \mathrm{H}$ spectrometers using peak matching to suitable reference peaks, depending on the technique used. Thin layer chromatography (TLC) was performed on precoated aluminium silica gel plates supplied by E. Merck, A. G. Darmstadt, Germany (Silica gel $60 \mathrm{~F}_{254}$, thickness $0.2 \mathrm{~mm}$, Art. 1.05554) or on precoated plastic silica gel plates supplied by Macherey-Nagel (Polygram ${ }^{\circledR}$ SIL G/UV ${ }_{254}$, thickness $0.25 \mathrm{~mm}$, Art. 805023 ) or on precoated glass plates supplied by Merck (Silica gel $60 \mathrm{~F}_{254}$, art. 1.05715). Visualisation was achieved by UV light and/or potassium permanganate stain. Flash column chromatography was performed using silica gel (Fluorochem, Silica gel 60, 40-63 $\mu$, Art. 02050017) or using a Biotage flash chromatography system. All glassware was oven dried $\left(100^{\circ} \mathrm{C}\right)$ overnight.

Light petroleum refers to the fraction boiling in the range 40 $60{ }^{\circ} \mathrm{C}$. Tetrahydrofuran was dried by refluxing with sodium metal and benzophenone until a deep purple colour persisted. Toluene, dichloromethane, diethyl ether and chloroform were all dried by refluxing with calcium hydride. All solvents were distilled and collected by dry syringe as required. Tetrahydrofuran for Stille coupling reactions was degassed by bubbling argon through the solvent for 30 minutes immediately before use. $n$-Butyllithium and $t$-butyllithium were titrated before use against 4-phenylbenzylidene benzylamine according to the method of Duhamel et al. ${ }^{37}$ Ethyl chloroformate $(99 \%)$ was stored under an atmosphere of argon once opened. 2,2Difluoro-1-( $N, N$-diethylcarbamoyloxy) vinyltributyltin was prepared according to the method of Howarth et al. ${ }^{21} 1-(N, N-$ Diethylcarbamoyloxy) ethene was prepared according to the method of Snieckus et $a .^{38}$ Copper(I) iodide was purified according to the method of Taylor et al. ${ }^{39}$ All other chemicals were used as received without any further purification.

\section{Ethyl 2-( $N, N$-diethylcarbamoyloxy)-3,3-difluoro-2-propenoate 8}

Ethyl chloroformate $(1 \mathrm{~mol}, 100 \mathrm{~mL})$ was added by syringe pump over one hour to a mixture of stannane $7^{21}(100 \mathrm{mmol}$, $40 \mathrm{~mL}$ ), palladium acetate (5 mmol, $0.64 \mathrm{~g})$, triphenylphosphine (20 mmol, $2.7 \mathrm{~g}$ ), and copper(I) iodide ( $5 \mathrm{mmol}, 1 \mathrm{~g}$ ) in THF $(350 \mathrm{~mL})$ at $60^{\circ} \mathrm{C}$. Stirring was maintained at this temperature for 2 hours; after cooling, the solvent was removed in vacuo and the resulting oil purified by filtration through a 
short column of silica ( $5 \%$ diethyl ether in light petroleum) to remove tributyltin chloride, followed by elution of the remainder of the material with diethyl ether. After concentration, the yellow oil was purified by Kugelrohr distillation to afford alkenoate 8 (17.6 g, 70\%, 98\% by GC) as a colourless oil; bp $70{ }^{\circ} \mathrm{C} / 0.1 \mathrm{~mm} \mathrm{Hg} ; R_{\mathrm{f}}(20 \%$ diethyl ether in light petroleum $)$ $0.3 ; v_{\max }($ film $) / \mathrm{cm}^{-1} 2982 \mathrm{~m} \quad(\mathrm{C}-\mathrm{H}), 2939 \mathrm{~m} \quad(\mathrm{C}-\mathrm{H}), 2879 \mathrm{~m}$ $(\mathrm{C}-\mathrm{H}), 1743 \mathrm{br}$ s $(\mathrm{C}=\mathrm{O}), 1343 \mathrm{~s}(\mathrm{C}-\mathrm{O}), 1307 \mathrm{~s}(\mathrm{C}-\mathrm{O}), 1191 \mathrm{~s}$ $(\mathrm{C}-\mathrm{O}) ; \delta_{\mathrm{H}}\left(300 \mathrm{MHz}, \mathrm{CDCl}_{3}\right) 4.27\left(2 \mathrm{H}, \mathrm{q}, J 7.1, \mathrm{OCH}_{2}\right), 3.39$ $3.29\left(4 \mathrm{H}, \mathrm{m}, \mathrm{NCH}_{2}\right), 1.29\left(3 \mathrm{H}, \mathrm{t}, J 7.1, \mathrm{OCH}_{2} \mathrm{CH}_{3}\right), 1.2(3 \mathrm{H}, \mathrm{t}$, $J$ 7.1, $\left.\mathrm{NCH}_{2} \mathrm{CH}_{3}\right), 1.18\left(3 \mathrm{H}, \mathrm{t}, J 7.1, \mathrm{NCH}_{2} \mathrm{CH}_{3}\right) ; \delta_{\mathrm{C}}(75 \mathrm{MHz}$, $\left.\mathrm{CDCl}_{3}\right) 160.7\left(\mathrm{t},{ }^{3} J_{\mathrm{C}-\mathrm{F}} 8.2\right), 159.5\left(\mathrm{dd},{ }^{1} J_{\mathrm{C}-\mathrm{F}} 305.7,299.5\right), 152.4$ $\left(\mathrm{dd},{ }^{4} J_{\mathrm{C}-\mathrm{F}} 3.1,2.0\right), 106.9\left(\mathrm{dd},{ }^{2} J_{\mathrm{C}-\mathrm{F}} 32.8,18.1\right), 61.6,42.7,42.1$, $13.8,13.6,13.0 ; \delta_{\mathrm{F}}\left(282 \mathrm{MHz}, \mathrm{CDCl}_{3}\right)-80.3\left(1 \mathrm{~F}, \mathrm{~d},{ }^{2} J_{\mathrm{F}-\mathrm{F}} 5.1\right)$, $-85.3\left(1 \mathrm{~F}, \mathrm{~d},{ }^{2} J_{\mathrm{F}-\mathrm{F}} 5.1\right)$; [HRMS (ES, $\left.[\mathrm{M}+\mathrm{Na}]^{+}\right)$Found: 274.0867. Calc for $\mathrm{C}_{10} \mathrm{H}_{15} \mathrm{NO}_{4} \mathrm{~F}_{2} \mathrm{Na}$ : 274.0861]; $\mathrm{m} / \mathrm{z}$ (CI) 269 $\left(100 \%,\left[\mathrm{M}+\mathrm{NH}_{4}\right]^{+}\right), 252\left(100 \%,[\mathrm{M}+\mathrm{H}]^{+}\right), 100(15), 74(68)$ 58 (10), 44 (13)and diene 11 (traces) $R_{\mathrm{f}}(20 \%$ diethyl ether in light petroleum) $0.14 ; \delta_{\mathrm{H}}\left(300 \mathrm{MHz}, \mathrm{CDCl}_{3}\right) 3.40-3.20(8 \mathrm{H}, \mathrm{m}$, $\left.\mathrm{NCH}_{2}\right), 1.22\left(12 \mathrm{H}, \mathrm{t}, J 7.1, \mathrm{NCH}_{2} \mathrm{CH}_{3}\right) ; \delta_{\mathrm{C}}\left(75 \mathrm{MHz}, \mathrm{CDCl}_{3}\right)$ $155.6,131.8\left(\mathrm{dd},{ }^{1} J_{\mathrm{C}-\mathrm{F}} 322.4,272.6\right), 115.9\left(\mathrm{dd},{ }^{2} J_{\mathrm{C}-\mathrm{F}} 65.4,64.1\right)$, $42.4,41.9,13.8,13.2 ; \delta_{\mathrm{F}}\left(282 \mathrm{MHz}, \mathrm{CDCl}_{3}\right)-91.9\left(2 \mathrm{~F}, \mathrm{~d},{ }^{2} J_{\mathrm{F}-\mathrm{F}}\right.$ 41.0), $-98.2\left(2 \mathrm{~F}, \mathrm{~d},{ }^{2} J_{\mathrm{F}-\mathrm{F}} 41.0\right) ; \mathrm{m} / z$ (CI) $357\left(100 \%,[\mathrm{M}+\mathrm{H}]^{+}\right.$, $100\left(40,\left[\mathrm{CONEt}_{2}\right]^{+}\right)$.

\section{Preparation of 8 via organozinc reagent}

2,2-Difluoro-1-( $N, N$-diethylcarbamoyloxy)ethene $(10 \mathrm{mmol}$, $1.80 \mathrm{~g})$ in THF $(5 \mathrm{~mL})$ was added over 15 minutes to a solution of tert-butyllithium $(10 \mathrm{mmol}, 6.0 \mathrm{~mL}$ of a $1.7 \mathrm{M}$ solution in pentane) in THF $(45 \mathrm{~mL})$ at $-78^{\circ} \mathrm{C}$. The deep blue solution was stirred for 60 minutes at $-78{ }^{\circ} \mathrm{C}$ before $\mathrm{ZnCl}_{2}(10 \mathrm{mmol}$, $10 \mathrm{~mL}$ of a $1 \mathrm{M}$ solution in THF) was added. The resulting solution was stirred for 90 minutes at $-78^{\circ} \mathrm{C}$ before being warmed to $50{ }^{\circ} \mathrm{C}$; during this time the colour of the solution changed from blue to yellow. $\mathrm{Pd}_{2} \mathrm{dba}_{3} \cdot \mathrm{CHCl}_{3}(0.2 \mathrm{mmol}, 0.2 \mathrm{~g})$ and $\mathrm{PPh}_{3}(0.8 \mathrm{mmol}, 1.05 \mathrm{~g})$ were added as a solution in THF $(2 \mathrm{~mL})$ in one portion, followed by ethyl chloroformate $(100$ mmol, $10 \mathrm{~mL}$ ) added over 60 minutes. The solution was stirred for 16 hours at $50{ }^{\circ} \mathrm{C}$ before being cooled to room temperature and being quenched with water $(20 \mathrm{~mL})$. The phases were separated and the aqueous layer extracted with ethyl acetate $(3 \times$ $20 \mathrm{~mL})$, the combined organic extracts were dried $\left(\mathrm{MgSO}_{4}\right)$, filtered and concentrated in vacuo to leave an oil which was purified by Kugelrohr distillation to yield $8(1.31 \mathrm{~g}, 52 \%)$ as a colourless oil.

Ethyl endo-2-( $N, N$-diethylcarbamoyloxy)-3,3-difluoro-7-oxabicyclo[2.2.1] hept-5-enyl-2-exo-carboxylate 12a, ethyl exo-2( $N, N$-diethylcarbamoyloxy)-3,3-difluoro-7-oxabicyclo[2.2.1]hept-5-enyl-2-endo-carboxylate $12 \mathrm{~b}$ and ethyl 4,4-difluoro-5hydroxy-2-oxo-5,7a-dihydro-4H-benzo[1,3]dioxole-3a-carboxylate 15

Tin(IV) chloride (15 mmol, $14.5 \mathrm{~mL}$ of a freshly-prepared $1.03 \mathrm{M}$ solution in DCM) was added slowly to a solution of alkenoate $8(60 \mathrm{mmol}, 15.1 \mathrm{~g})$ and furan $(120 \mathrm{mmol}, 8.8 \mathrm{~mL})$ in DCM $(81 \mathrm{~mL})$ at $0{ }^{\circ} \mathrm{C}$ under an atmosphere of nitrogen. The solution was allowed to warm to room temperature and was stirred for 17 hours. The mixture was diluted with water $(20 \mathrm{~mL})$ and extracted with ethyl acetate $(3 \times 20 \mathrm{~mL})$. The combined organic extracts were dried $\left(\mathrm{MgSO}_{4}\right)$ and concentrated in vacuo to afford an orange solid (22.3 g) which recrystallised from methanol to afford endo 12a as cubes $(7.3 \mathrm{~g}$, $38 \%)$, mp $110-113{ }^{\circ} \mathrm{C} ; R_{\mathrm{f}}(20 \%$ diethyl ether in light petroleum $)$ 0.13; (Found: $\mathrm{C}, 52.68 ; \mathrm{H}, 5.81 ; \mathrm{N}, 4.46 ; \mathrm{C}_{14} \mathrm{H}_{19} \mathrm{~F}_{2} \mathrm{NO}_{5}$ requires: C $52.66 ; \mathrm{H}, 6.00 ; \mathrm{N}, 4.39 \%) ; v_{\max }($ film $) / \mathrm{cm}^{-1} 2985 \mathrm{w}(\mathrm{C}-\mathrm{H})$, $1750 \mathrm{~s}(\mathrm{C}=\mathrm{O}), 1709 \mathrm{~s}(\mathrm{C}=\mathrm{O}), 1291 \mathrm{~s}(\mathrm{C}-\mathrm{O}), 1172 \mathrm{~s}(\mathrm{C}-\mathrm{O}), 1122 \mathrm{~s}$ $(\mathrm{C}-\mathrm{O}) ; \delta_{\mathrm{H}}\left(300 \mathrm{MHz}, \mathrm{CDCl}_{3}\right) 6.93\left(1 \mathrm{H}, \mathrm{dd}, J 5.9,1.7, H_{\mathrm{a}} \mathrm{C}=\right.$ $\left.\mathrm{CH}_{\mathrm{b}}\right), 6.37\left(1 \mathrm{H}, \mathrm{d}, J 5.9, \mathrm{H}_{\mathrm{a}} \mathrm{C}=\mathrm{C} H_{\mathrm{b}}\right), 5.30-5.28(1 \mathrm{H}, \mathrm{m}, \mathrm{CHO})$ $4.79\left(1 \mathrm{H}, \mathrm{dd}, J, 1.7,{ }^{3} J_{\mathrm{H}-\mathrm{F}} 4.7, \mathrm{CHOCF}_{2}\right), 4.28-4.08(2 \mathrm{H}, \mathrm{m}$,
$\left.\mathrm{OCH}_{2}\right), 3.48-3.23\left(4 \mathrm{H}, \mathrm{m}, \mathrm{NCH}_{2}\right), 1.25-1.18\left(6 \mathrm{H}, \mathrm{m}, \mathrm{OCH}_{2}-\right.$ $\left.\mathrm{CH}_{3}, \mathrm{NCH}_{2} \mathrm{CH}_{3}\right), 1.13\left(3 \mathrm{H}, \mathrm{t}, J 7.2, \mathrm{NCH}_{2} \mathrm{CH}_{3}\right) ; \delta_{\mathrm{C}}(75 \mathrm{MHz}$, $\left.\mathrm{CDCl}_{3}\right) 165.5,154.1,138.6\left(\mathrm{t},{ }^{4} J_{\mathrm{C}-\mathrm{F}} 1.4\right), 130.1\left(\mathrm{~d},{ }^{3} J_{\mathrm{C}-\mathrm{F}} 4.5\right)$, $123.5\left(\mathrm{dd},{ }^{1} J_{\mathrm{C}-\mathrm{F}} 281.0,261.0\right), 85.0\left(\mathrm{~d},{ }^{3} J_{\mathrm{C}-\mathrm{F}} 4.0\right), 82.8\left(\mathrm{dd},{ }^{2} J_{\mathrm{C}-\mathrm{F}}\right.$ 27.7, 20.1), 81.2 (dd, $\left.{ }^{2} J_{\text {C-F }} 29.1,26.8\right), 62.1,42.4,42.7,14.1$, $14.0,13.6 ; \delta_{\mathrm{F}}\left(282 \mathrm{MHz}, \mathrm{CDCl}_{3}\right)-103.5\left(1 \mathrm{~F}, \mathrm{~d},{ }^{2} J_{\mathrm{F}-\mathrm{F}} 225.1\right)$, $-113.6\left(1 \mathrm{~F}, \mathrm{dd},{ }^{2} J_{\mathrm{F}-\mathrm{F}} 225.1,{ }^{3} J_{\mathrm{F}-\mathrm{H}} 4.7\right)$ [HRMS (FAB, M) Found: 320.131005 . Calc. for $\mathrm{C}_{14} \mathrm{H}_{19} \mathrm{~F}_{2} \mathrm{NO}_{5}$ : 320.130955]; $\mathrm{m} / \mathrm{z}$ (FAB) $342\left(84 \%\left[\mathrm{M}+\mathrm{Na}^{+}\right), 320(100), 274(13), 252(8)\right.$, 206(14), 176(16), 154(9), 137(9), 100 (87). The mother liquor was concentrated in vacuo and the resulting orange oil purified by column chromatography $(20 \%$ diethyl ether in light petroleum) to afford more endo adduct 12a (2.63 g, 14\%), and exo adduct 12b $(2.64 \mathrm{~g}, 14 \%)$ as cubes $\mathrm{mp} 68-70{ }^{\circ} \mathrm{C} ; R_{\mathrm{f}}(20 \%$ diethyl ether in light petroleum) 0.1 (Found: $\mathrm{C}, 52.81 ; \mathrm{H}, 6.08$; $\mathrm{N}, 4.44 ; \mathrm{C}_{14} \mathrm{H}_{19} \mathrm{~F}_{2} \mathrm{NO}_{5}$ requires: $\mathrm{C} 52.66 ; \mathrm{H}, 6.00 ; \mathrm{N}, 4.39 \%$ ); $v_{\max }(\mathrm{Nujol}) / \mathrm{cm}^{-1} 2985 \mathrm{~m}(\mathrm{C}-\mathrm{H}), 2939 \mathrm{~m}(\mathrm{C}-\mathrm{H}), 1760 \mathrm{~s}(\mathrm{C}=\mathrm{O})$, $1715 \mathrm{~s}(\mathrm{C}=\mathrm{O}), 1428 \mathrm{~m}(\mathrm{C}-\mathrm{C}), 1278 \mathrm{~s}(\mathrm{C}-\mathrm{O}), 1170 \mathrm{~s}(\mathrm{C}-\mathrm{O}) ; \delta_{\mathrm{H}}(300$ $\left.\mathrm{MHz}, \mathrm{CDCl}_{3}\right) 6.47-5.52(2 \mathrm{H}, \mathrm{m}, \mathrm{HC}=\mathrm{CH}), 5.70(1 \mathrm{H}, \mathrm{s}, \mathrm{CHO})$, $4.81\left(1 \mathrm{H}, \mathrm{dd},{ }^{3} J_{\mathrm{H}-\mathrm{F}} 5.5, J 0.7, \mathrm{CHOCF}_{2}\right), 4.35-4.19(2 \mathrm{H}, \mathrm{m}$, $\left.\mathrm{OCH}_{2}\right), 3.29-3.14\left(4 \mathrm{H}, \mathrm{m}, \mathrm{NCH}_{2}\right), 1.26(3 \mathrm{H}, \mathrm{t}, J 7.2$, $\left.\mathrm{OCH}_{2} \mathrm{CH}_{3}\right), 1.10\left(3 \mathrm{H}, \mathrm{t}, J 7.1, \mathrm{NCH}_{2} \mathrm{CH}_{3}\right), 1.08(3 \mathrm{H}, \mathrm{t}, J 7.1$, $\left.\mathrm{NCH}_{2} \mathrm{CH}_{3}\right) ; \delta_{\mathrm{C}}\left(75 \mathrm{MHz}, \mathrm{CDCl}_{3}\right) 166.0\left(\mathrm{~d},{ }^{3} J_{\mathrm{C}-\mathrm{F}} 5.1\right), 153.3(\mathrm{~d}$, $\left.{ }^{4} J_{\mathrm{C}-\mathrm{F}} 1.1\right), 137.6\left(\mathrm{dd},{ }^{4} J_{\mathrm{C}-\mathrm{F}} 2.3,1.1\right), 133.2\left(\mathrm{dd},{ }^{3} J_{\mathrm{C}-\mathrm{F}} 4.5,1.1\right)$, $122.6\left(\mathrm{dd},{ }^{1} J_{\mathrm{C}-\mathrm{F}} 276.9,263.4\right), 84.0\left(\mathrm{~d},{ }^{3} J_{\mathrm{C}-\mathrm{F}} 2.3\right), 80.2\left(\mathrm{t},{ }^{2} J_{\mathrm{C}-\mathrm{F}}\right.$ 27.1), $79.8\left(\mathrm{~d},{ }^{2} J_{\mathrm{C}-\mathrm{F}} 27.1\right), 62.3,42.4,42.1,14.1,14.0,13.5$; $\delta_{\mathrm{F}}\left(282 \mathrm{MHz}, \mathrm{CDCl}_{3}\right)-107.2\left(1 \mathrm{~F}, \mathrm{~d},{ }^{2} J_{\mathrm{C}-\mathrm{F}} 225.1\right),-109.3(1 \mathrm{~F}$, $\left.\mathrm{dd},{ }^{2} J_{\mathrm{F}-\mathrm{F}} 225.1,{ }^{3} J_{\mathrm{F}-\mathrm{H}} 5.1\right)$ [HRMS (ES, $\left.[\mathrm{M}+\mathrm{Na}]^{+}\right)$Found: 342.1117. Calc. for $\mathrm{C}_{14} \mathrm{H}_{19} \mathrm{~F}_{2} \mathrm{NO}_{5} \mathrm{Na} 342.1129$ ]; $\mathrm{m} / z$ (ES) 342 $\left(100 \%,[\mathrm{M}+\mathrm{Na}]^{+}\right)$and cyclohexenol $15(0.4 \mathrm{~g}, 2 \%)$ as cubes; $\mathrm{mp} 61-62{ }^{\circ} \mathrm{C} ; R_{\mathrm{f}}(20 \%$ diethyl ether in light petroleum $) 0.05$ (Found: $\mathrm{C}, 45.66 ; \mathrm{H}, 3.70 ; \mathrm{C}_{10} \mathrm{H}_{10} \mathrm{~F}_{2} \mathrm{O}_{6}$ requires: $\mathrm{C}, 45.46 ; \mathrm{H}$, $3.82 \%) ; v_{\max }(\mathrm{film}) / \mathrm{cm}^{-1} 3538 \mathrm{~m}$ br $(\mathrm{OH}), 2996 \mathrm{~m}(\mathrm{C}-\mathrm{H}), 1846 \mathrm{~m}$ $(\mathrm{C}=\mathrm{O}), 1750 \mathrm{~m} \quad(\mathrm{C}=\mathrm{O}), 1473 \mathrm{w} \quad(\mathrm{C}-\mathrm{C}), 1315 \mathrm{~m} \quad(\mathrm{C}-\mathrm{O}), 1229 \mathrm{~m}$ $(\mathrm{C}-\mathrm{O}), 1118 \mathrm{~m}(\mathrm{C}-\mathrm{O}) ; \delta_{\mathrm{H}}\left(300 \mathrm{MHz}, \mathrm{CDCl}_{3}\right) 6.15-6.09(1 \mathrm{H}, \mathrm{m}$, $\left.H_{\mathrm{a}} \mathrm{C}=\mathrm{CH}_{\mathrm{b}}\right), 5.96-5.91\left(1 \mathrm{H}, \mathrm{m}, \mathrm{H}_{\mathrm{a}} \mathrm{C}=\mathrm{CH}_{\mathrm{b}}\right), 5.48(1 \mathrm{H}, \mathrm{d}, J 4.0$, $\mathrm{CHOCO}), 4.68-4.61(1 \mathrm{H}, \mathrm{m}, \mathrm{C} H \mathrm{OH}), 4.42-4.32(2 \mathrm{H}, \mathrm{m}$, $\left.\mathrm{OCH}_{2}\right), 3.66(1 \mathrm{H}$, br. s, $\mathrm{OH}), 1.34\left(3 \mathrm{H}, \mathrm{t}, J 7.2, \mathrm{OCH}_{2} \mathrm{CH}_{3}\right)$; $\delta_{\mathrm{C}}\left(75 \mathrm{MHz}, \mathrm{CDCl}_{3}\right) 164.2\left(\mathrm{~d},{ }^{4} J_{\mathrm{C}-\mathrm{F}} 1.7\right), 151.3,133.5\left(\mathrm{dd},{ }^{3} J_{\mathrm{C}-\mathrm{F}}\right.$ $5.9,1.4), 122.0\left(\mathrm{~d},{ }^{4} J_{\mathrm{C}-\mathrm{F}} 1.1\right), 116.6\left(\mathrm{dd},{ }^{1} J_{\mathrm{C}-\mathrm{F}} 256.6,250.9\right), 80.6$ $\left(\mathrm{dd},{ }^{2} J_{\mathrm{C}-\mathrm{F}} 33.1,23.5\right), 76.8\left(\mathrm{~d},{ }^{3} J_{\mathrm{C}-\mathrm{F}} 2.3\right), 65.2\left(\mathrm{dd},{ }^{2} J_{\mathrm{C}-\mathrm{F}} 27.1\right.$, 20.9), 64.2, 13.7; $\delta_{\mathrm{F}}\left(282 \mathrm{MHz}, \mathrm{CDCl}_{3}\right)-115.3\left(1 \mathrm{~F}, \mathrm{~d},{ }^{2} J_{\mathrm{F}-\mathrm{F}}\right.$ 261.5), $-125.9\left(1 \mathrm{~F}, \mathrm{dd},{ }^{2} J_{\mathrm{F}-\mathrm{F}} 261.5,{ }^{3} J_{\mathrm{F}-\mathrm{H}} 13.4\right) ; \mathrm{m} / z$ (CI) 287 $\left(100 \%,[\mathrm{M}+\mathrm{Na}]^{+}\right)$.

Ethyl-2-exo-( $N, N$-diethylcarbamoyloxy)-3,3-difluoro-1-methyl7-oxabicyclo[2.2.1]hept-5-enyl-2-endo-carboxylate 13a and ethyl-2-endo-( $N, N$-diethylcarbamoyloxy-3,3-difluoro)-1-methyl7-oxabicyclo[2.2.1]hept-5-enyl-2-exo-carboxylate 13b

Prepared as for 12a and $\mathbf{1 2 b}$ from alkenoate $\mathbf{8}(10 \mathrm{mmol}, 2.51 \mathrm{~g})$, 2-methylfuran (20 mmol, $2.4 \mathrm{~mL})$; tin(Iv) chloride ( $2.5 \mathrm{mmol}$, $2.4 \mathrm{~mL}$ of a $1.03 \mathrm{M}$ solution in DCM) in DCM $(15 \mathrm{~mL})$ was added at $0{ }^{\circ} \mathrm{C}$. After stirring for 30 minutes at room temperature, the mixture was diluted with water $(10 \mathrm{~mL})$ and extracted with ethyl acetate $(3 \times 20 \mathrm{~mL})$. The combined organic extracts were dried $\left(\mathrm{MgSO}_{4}\right)$ and concentrated in vacuo to leave a yellow oil which was purified by column chromatography $(50 \%$ diethyl ether in light petroleum) to afford an inseparable mixture of adducts 13a and 13b $(3.1 \mathrm{~g}, 93 \%)$ as a colourless oil; $R_{\mathrm{f}}(50 \%$ diethyl ether in light petroleum) $0.45 ; v_{\max }($ film $) / \mathrm{cm}^{-1} 2981 \mathrm{~s}$ $(\mathrm{C}-\mathrm{H}), 2939 \mathrm{~s}(\mathrm{C}-\mathrm{H}), 1756 \mathrm{~s}(\mathrm{C}=\mathrm{O}), 1717 \mathrm{~s}(\mathrm{C}=\mathrm{O}), 1478 \mathrm{~s}(\mathrm{C}-\mathrm{C})$, $1427 \mathrm{~s}(\mathrm{C}-\mathrm{C}), 1168 \mathrm{~s}(\mathrm{C}-\mathrm{O}), 1076 \mathrm{~s}(\mathrm{C}-\mathrm{O}) ; \delta_{\mathrm{H}}\left(300 \mathrm{MHz}, \mathrm{CDCl}_{3}\right)$ 6.44-6.26 (4H, env., $\mathrm{HC}=\mathrm{CH}), 4.76\left(1 \mathrm{H}, \mathrm{dd},{ }^{3} J_{\mathrm{H}-\mathrm{F}} 4.2,2.0\right.$, $\left.\mathrm{CHOCF}_{2}\right), 4.64\left(1 \mathrm{H}, \mathrm{dt},{ }^{3} J_{\mathrm{H}-\mathrm{F}} 5.9,{ }^{3} J_{\mathrm{H}-\mathrm{F}} 2.0, \mathrm{CHOCF}_{2}\right), 4.21$ $\left(2 \mathrm{H}, \mathrm{q}, J 7.1, \mathrm{OCH}_{2}\right), 4.10\left(2 \mathrm{H}, \mathrm{q}, J 7.1, \mathrm{OCH}_{2}\right), 3.41-3.11(8 \mathrm{H}$, env., $\left.\mathrm{NCH}_{2}\right), 1.70\left(3 \mathrm{H}, \mathrm{s}, \mathrm{C}\left(\mathrm{CH}_{3}\right) \mathrm{O}\right), 1.53\left(3 \mathrm{H}, \mathrm{s}, \mathrm{C}\left(\mathrm{CH}_{3}\right) \mathrm{O}\right)$, 1.24-1.03 (18H, env., $\left.\mathrm{OCH}_{2} \mathrm{CH}_{3}, \mathrm{NCH}_{2} \mathrm{CH}_{3}\right) ; \delta_{\mathrm{C}}(75 \mathrm{MHz}$, $\left.\mathrm{CDCl}_{3}\right) 165.8,165.0,154.2,153.2,141.0,140.4,133.2\left(\mathrm{~d},{ }^{3} J_{\mathrm{C}-\mathrm{F}}\right.$ 4.0), $133.0\left(\mathrm{~d},{ }^{3} J_{\mathrm{C}-\mathrm{F}} 5.1\right), 123.8\left(\mathrm{t},{ }^{1} J_{\mathrm{C}-\mathrm{F}} 271.6\right), 123.3\left(\mathrm{dd},{ }^{1} J_{\mathrm{C}-\mathrm{F}}\right.$ 
275.2, 269.6), $92.0\left(\mathrm{~d},{ }^{3} J_{\mathrm{C}-\mathrm{F}} 4.0\right), 91.4,82.2\left(\mathrm{t},{ }^{2} J_{\mathrm{C}-\mathrm{F}} 16.9\right), 81.0(\mathrm{t}$ $\left.{ }^{2} J_{\mathrm{C}-\mathrm{F}} 27.4\right), 80.8\left(\mathrm{t},{ }^{2} J_{\mathrm{C}-\mathrm{F}} 28.0\right), 61.7,61.5,42.5,42.3,42.2,42.0$, $16.4,14.9,14.1,14.0,13.9,13.8,13.5,13.4 ; \delta_{\mathrm{F}}(282 \mathrm{MHz}$ $\left.\mathrm{CDCl}_{3}\right)-101.0$ to -103.9 (br m), $-104.5\left(\mathrm{~d},{ }^{2} J_{\mathrm{F}-\mathrm{F}} 222.5\right)$, -108.0 to -110.0 (br m, overlapping. -108.9 (dd, ${ }^{2} J_{\mathrm{F}-\mathrm{F}} 222.5$, ${ }^{3} J_{\mathrm{F}-\mathrm{H}}$ 5.9) [HRMS (ES, [M + Na] ${ }^{+}$) Found: 356.1287. Calc. for $\left.\mathrm{C}_{15} \mathrm{H}_{21} \mathrm{~F}_{2} \mathrm{NO}_{5} \mathrm{Na} 356.1285\right] ; \mathrm{m} / z$ (ES) $356\left(100 \%[\mathrm{M}+\mathrm{Na}]^{+}\right)$. The ${ }^{1} \mathrm{H}$ NMR spectrum of the mixture could not be assigned fully because of overlap between stereoisomers. The ${ }^{19} \mathrm{~F}$ NMR spectrum could not be integrated because of the breadth of the signals for one stereoisomer. Exo-cycloadduct $\mathbf{1 3 b}$ crystallised from this mixture $\mathrm{mp} 52-53{ }^{\circ} \mathrm{C}$ (Found: $\mathrm{C}, 53.89 ; \mathrm{H}, 6.31 ; \mathrm{N}$, 4.13; $\mathrm{C}_{14} \mathrm{H}_{19} \mathrm{~F}_{2} \mathrm{NO}_{5}$ requires: $\mathrm{C} 54.05 ; \mathrm{H}, 6.35 ; \mathrm{N}, 4.20 \%$; $\delta_{\mathrm{H}}\left(300 \mathrm{MHz}, \mathrm{CDCl}_{3}\right) 6.58-6.47(1 \mathrm{H}, \mathrm{m},=\mathrm{CHCHO}), 6.34(1 \mathrm{H}$ $\left.\mathrm{d}, J 5.5,=\mathrm{CHC}\left(\mathrm{CH}_{3}\right) \mathrm{O}\right), 4.86-4.82(1 \mathrm{H}, \mathrm{m},=\mathrm{CHCHO}), 4.30$ $\left(2 \mathrm{H}, \mathrm{q}, J\right.$ 7.0, $\left.\mathrm{OCH}_{2} \mathrm{CH}_{3}\right), 3.38-3.15\left(4 \mathrm{H}, \mathrm{m}, \mathrm{NCH}_{2} \mathrm{CH}_{3}\right), 1.79$ $\left(3 \mathrm{H}, \mathrm{s}, \mathrm{C}\left(\mathrm{CH}_{3}\right) \mathrm{O}\right), 1.30\left(3 \mathrm{H}, \mathrm{t}, J 7.0, \mathrm{OCH}_{2} \mathrm{CH}_{3}\right), 1.13(6 \mathrm{H}, \mathrm{t}$, $J$ 7.0, $\left.\mathrm{NCH}_{2} \mathrm{CH}_{3}\right) ; \delta_{\mathrm{C}}\left(75 \mathrm{MHz}, \mathrm{CDCl}_{3}\right)$ 165.0, 153.2, 141.0, $133.0,123.8\left(\mathrm{t},{ }^{1} J_{\mathrm{C}-\mathrm{F}} 271.6\right), 91.4,80.8\left(\mathrm{t},{ }^{2} J_{\mathrm{C}-\mathrm{F}} 28.0\right), 61.7,42.3$, $42.0,16.4,14.0,13.9,13.4 ; \delta_{\mathrm{F}}\left(282 \mathrm{MHz}, \mathrm{CDCl}_{3}\right)-101.0$ to -103.9 (br m), -108.0 to -110.0 (br m, overlapping).

Ethyl-2-endo-( $N, N$-diethylcarbamoyloxy)-3,3-difluoro-1,6-dimethyl-7-oxabicyclo[2.2.1]hept-5-enyl-2-exo-carboxylate 14b

Tin (IV) chloride $(8 \mathrm{mmol}, 0.94 \mathrm{~mL})$, was added slowly to a solution of alkenoate $\mathbf{8}(32 \mathrm{mmol}, 8.0 \mathrm{~g})$ and 2,3-methylfuran $(64 \mathrm{mmol}, 6.7 \mathrm{~mL})$ in DCM $(13 \mathrm{~mL})$ at $0{ }^{\circ} \mathrm{C}$ under an atmosphere of nitrogen. The solution was stirred for 30 minutes at $0{ }^{\circ} \mathrm{C}$. The mixture was diluted with water $(10 \mathrm{~mL})$ and extracted with ethyl acetate $(3 \times 20 \mathrm{~mL})$. The combined organic extracts were dried $\left(\mathrm{MgSO}_{4}\right)$ and concentrated in vacuo to leave an orange oil which was purified by column chromatography $(40 \%$ diethyl ether in light petroleum) to afford a mixture of endo and exo $(1: 1.4)$ isomers as a yellow oil from which exo $\mathbf{1 4 b}$ crystallized. The mixture was washed with hexane to yield exo $\mathbf{1 4 b}$ $(5.9 \mathrm{~g}, 53 \%)$ as cubes, $R_{\mathrm{f}}(20 \%$ diethyl ether in light petroleum $)$ $0.11 ; \mathrm{mp} 84^{\circ} \mathrm{C}$ (Found: C, 55.12; H, 6.54, N 3.99; $\mathrm{C}_{16} \mathrm{H}_{23} \mathrm{~F}_{2} \mathrm{O}_{5} \mathrm{~N}$ requires: C, 55.32; H, $6.67 \mathrm{~N} \mathrm{4.03 \% );} v_{\max }($ film $) / \mathrm{cm}^{-1} 2977 \mathrm{w}$ $(\mathrm{C}-\mathrm{H}), 2939 \mathrm{w}(\mathrm{C}-\mathrm{H}), 1755 \mathrm{~s}(\mathrm{C}=\mathrm{O}), 1713 \mathrm{~s}(\mathrm{C}=\mathrm{O}), 1479 \mathrm{~m}(\mathrm{C}-\mathrm{C})$, $1424 \mathrm{~m}(\mathrm{C}-\mathrm{C}), 1281 \mathrm{~s}(\mathrm{C}-\mathrm{O}) ; \delta_{\mathrm{H}}\left(400 \mathrm{MHz}, \mathrm{C}_{6} \mathrm{D}_{6}, 348 \mathrm{~K}\right) 5.87$ $(1 \mathrm{H}, \mathrm{s},=\mathrm{CH}), 4.55\left(1 \mathrm{H}, \mathrm{d}, J 6.2, \mathrm{CHOCF}_{2}\right), 4.36-4.24(2 \mathrm{H}, \mathrm{m}$, $\left.\mathrm{OCH}_{2}\right), 3.28-3.14\left(4 \mathrm{H}, \mathrm{m}, \mathrm{NCH}_{2}\right), 2.15\left(3 \mathrm{H}, \mathrm{s},=\mathrm{CCH}_{3}\right), 1.80$ $\left(3 \mathrm{H}, \mathrm{s}, \mathrm{C}\left(\mathrm{CH}_{3}\right) \mathrm{O}\right), 1.23\left(3 \mathrm{H}, \mathrm{t}, J 7.1, \mathrm{OCH}_{2} \mathrm{CH}_{3}\right), 1.13(6 \mathrm{H}, \mathrm{t}$, $J$ 7.1, $\left.\mathrm{NCH}_{2} \mathrm{CH}_{3}\right) ; \delta_{\mathrm{C}}\left(75 \mathrm{MHz}, \mathrm{CDCl}_{3}\right) 165.1,153.6,150.5$, $126.1,123.8\left(\mathrm{t}, J_{\mathrm{C}-\mathrm{F}} 270.6\right), 92.2,82.1\left(\mathrm{t},{ }^{2} J_{\mathrm{C}-\mathrm{F}} 19.7\right), 79.4\left(\mathrm{t},{ }^{2} J_{\mathrm{C}-\mathrm{F}}\right.$ 27.5), 61.7, 42.4, 42.1, 14.9, 14.0, 13.7, 13.3, 13.1; $\delta_{\mathrm{F}}(376 \mathrm{MHz}$ $\left.\mathrm{C}_{6} \mathrm{D}_{6}, 348 \mathrm{~K}\right)-104.8\left(\mathrm{~d}, J_{\mathrm{F}-\mathrm{F}} 221.1\right),-108.1\left(\mathrm{~d}, J_{\mathrm{F}-\mathrm{F}} 221.1\right) ; \mathrm{m} / \mathrm{z}$ (ES) $348\left(34 \%, \mathrm{M}^{+}\right), 328$ (100), 282 (67), 252 (38): and a mixture of a 14a and 14b as a yellow oil $(3.2 \mathrm{~g}, 29 \%)$. Distinct signals arising from endo 14a could also be observed: $\delta_{\mathrm{H}}(300 \mathrm{MHz}$, $\left.\left.\mathrm{CDCl}_{3}\right) 6.16(1 \mathrm{H}, \mathrm{s},=\mathrm{CH}), 4.58-4.56(1 \mathrm{H}, \mathrm{m}, \mathrm{CHOCF})_{2}\right), 4.15$ $\left(2 \mathrm{H}, \mathrm{q}, J 7.2, \mathrm{OCH}_{2}\right) ; \delta_{\mathrm{F}}\left(282 \mathrm{MHz}, \mathrm{CDCl}_{3}\right)-105.9\left(\mathrm{dd},{ }^{2} J_{\mathrm{F}-\mathrm{F}}\right.$ $\left.221.3,{ }^{3} J_{\mathrm{F}-\mathrm{H}} 5.1\right),-108.2\left(\mathrm{~d},{ }^{2} J_{\mathrm{F}-\mathrm{F}} 221.3\right)$. The ${ }^{19} \mathrm{~F}$ NMR spectrum could not be integrated because the signals were overlapped with the broad signals for $\mathbf{1 4 b}$.

\section{Preparation of ethyl-2-( $N, N$-diethylcarbamoyloxy)-2-propenoate 16}

1-( $N, N$-Diethylcarbamoyloxy) vinyltributyltin. tert-Butyllithium ( $23 \mathrm{mmol}, 15.5 \mathrm{~mL}$ of a $1.5 \mathrm{M}$ solution in pentane) was added slowly to a solution of 1 -( $N, N$-diethylcarbamoyloxy)ethene $^{37}(21 \mathrm{mmol}, 3 \mathrm{~g})$ in THF $(120 \mathrm{~mL})$ at $-78^{\circ} \mathrm{C}$. After stirring for 60 minutes at this temperature, tributyltin chloride (23 mmol, $6.3 \mathrm{~mL}$ ) was added and stirring maintained for 60 minutes at $-78{ }^{\circ} \mathrm{C}$, before saturated aqueous ammonium chloride $(50 \mathrm{~mL})$ was added. The phases were separated and the aqueous phase was extracted with diethyl ether $(3 \times 50 \mathrm{~mL})$. The combined organic extracts were dried $\left(\mathrm{MgSO}_{4}\right)$ filtered and concentrated in vacuo and the resulting oil was purified by column chromatography ( $5 \%$ diethyl ether in light petroleum) to yield 1-( $N, N$-diethylcarbamoyloxy)vinyl tributyltin as a colourless oil $(6.72 \mathrm{~g}, 74 \%) ; R_{\mathrm{f}}(5 \%$ diethyl ether in light petroleum) $0.71 ; v_{\max }(\mathrm{film}) / \mathrm{cm}^{-1} 2956 \mathrm{~s}(\mathrm{C}-\mathrm{H}), 2921 \mathrm{~s}(\mathrm{C}-\mathrm{H}), 2871 \mathrm{~s}(\mathrm{C}-\mathrm{H})$, $2859 \mathrm{~s}(\mathrm{C}-\mathrm{H}), 1697 \mathrm{~s}$ br $(\mathrm{C}=\mathrm{O}), 1474 \mathrm{~s}(\mathrm{C}-\mathrm{C}), 1459 \mathrm{~s}(\mathrm{C}-\mathrm{C}), 1427 \mathrm{~s}$ $(\mathrm{C}-\mathrm{C}), 1279 \mathrm{~s}(\mathrm{C}-\mathrm{O}), 864 \mathrm{~m}\left(\mathrm{C}=\mathrm{CH}_{2}\right) ; \delta_{\mathrm{H}}\left(300 \mathrm{MHz}, \mathrm{CDCl}_{3}\right)$ $5.35\left(1 \mathrm{H}, \mathrm{s},=\mathrm{CH}_{\mathrm{a}} \mathrm{H}_{\mathrm{b}}\right), 4.57\left(1 \mathrm{H}, \mathrm{s},=\mathrm{CH}_{\mathrm{a}} H_{\mathrm{b}}\right), 3.30-3.28(4 \mathrm{H}, \mathrm{m}$, $\left.\mathrm{NCH}_{2}\right), 1.54-1.42\left(6 \mathrm{H}, \mathrm{m}, \mathrm{SnCH}_{2}\right), 1.37-1.24(6 \mathrm{H}, \mathrm{m}$, $\left.\mathrm{NCH}_{2} \mathrm{CH}_{3}\right), 1.17-1.10\left(6 \mathrm{H}, \mathrm{m}, \mathrm{CH}_{2} \mathrm{CH}_{2} \mathrm{CH}_{3}\right), 0.95-0.89(9 \mathrm{H}$, $\left.\mathrm{m}, \mathrm{CH}_{2} \mathrm{CH}_{2} \mathrm{CH}_{3}\right), 0.87\left(6 \mathrm{H}, \mathrm{t}, J 7.2, \mathrm{SnCH}_{2} \mathrm{CH}_{2}\right) ; \delta_{\mathrm{C}}(75 \mathrm{MHz}$, $\mathrm{CDCl}_{3}$ ) 164.4, 155.0, 108.6, 41.9, 41.4, 29.1, 27.4, 14.2, 13.8, 13.5, 12.1 [HRMS (ES, $[\mathrm{M}+\mathrm{Na}]^{+}$) Found: 456.1901. Calc. for $\left.\mathrm{C}_{19} \mathrm{H}_{39} \mathrm{NO}_{2}{ }^{119} \mathrm{SnNa}: 456.1900\right] ; \mathrm{m} / \mathrm{z}$ (EI) $432\left(6 \% \mathrm{M}^{+}\right), 376$ (100), 350 (40), 236 (28), 177 (17), 131 (17), 100 (8), 69 (39).

Ethyl-2-( $N, N$-diethylcarbamoyloxy)-2-propenoate 16. Was prepared as for $\mathbf{8}$ from 1-(N,N-diethylcarbamoyloxy)vinyltributyltin (18 mmol, $7.8 \mathrm{~g})$, ethyl chloroformate (180 mmol, 17 $\mathrm{mL})$, palladium acetate $(0.5 \mathrm{mmol}, 0.11 \mathrm{~g})$, triphenylphosphine $(1.8 \mathrm{mmol}, 0.5 \mathrm{~g})$, and copper(I) iodide $(0.1 \mathrm{mmol}, 0.2 \mathrm{~g})$ in THF $(60 \mathrm{~mL})$. The resulting oil was purified by column chromatography ( $20 \%$ diethyl ether in light petroleum) to afford alkenoate $16\left(2.3 \mathrm{~g}, 60 \%, 100 \%\right.$ by GC) as a colourless oil; $R_{\mathrm{f}}$ $\left(20 \%\right.$ diethyl ether in light petroleum) $0.15 ; v_{\max }(\mathrm{film}) / \mathrm{cm}^{-1}$ $2979 \mathrm{~m}(\mathrm{C}-\mathrm{H}), 1722 \mathrm{~s}$ br $(\mathrm{C}=\mathrm{O}), 1651 \mathrm{~m}(\mathrm{C}=\mathrm{C}), 1426 \mathrm{~m}(\mathrm{C}-\mathrm{C})$, $1380 \mathrm{~m}(\mathrm{C}-\mathrm{O}), 1304 \mathrm{~s}(\mathrm{C}-\mathrm{O}), 1272 \mathrm{~s}(\mathrm{C}-\mathrm{O}), 1150 \mathrm{~s}(\mathrm{C}-\mathrm{O}) ; \delta_{\mathrm{H}}(300$ $\left.\mathrm{MHz}, \mathrm{CDCl}_{3}\right) 5.93\left(1 \mathrm{H}, \mathrm{d}, J 1.2,=\mathrm{CH}_{\mathrm{a}} \mathrm{H}_{\mathrm{b}}\right), 5.40(1 \mathrm{H}, \mathrm{d}, J 1.2,=$ $\left.\mathrm{CH}_{\mathrm{a}} H_{\mathrm{b}}\right), 4.22\left(2 \mathrm{H}, \mathrm{q}, J 7.2, \mathrm{OCH}_{2}\right), 3.38-3.28\left(4 \mathrm{H}, \mathrm{m}, \mathrm{NCH}_{2}\right)$, $1.28\left(3 \mathrm{H}, \mathrm{t}, J 7.2, \mathrm{OCH}_{2} \mathrm{CH}_{3}\right), 1.20\left(3 \mathrm{H}, \mathrm{t}, J 7.2, \mathrm{NCH}_{2} \mathrm{CH}_{3}\right)$, $1.16\left(3 \mathrm{H}, \mathrm{t}, J 7.2, \mathrm{NCH}_{2} \mathrm{CH}_{3}\right) ; \delta_{\mathrm{C}}\left(75 \mathrm{MHz}, \mathrm{CDCl}_{3}\right)$ 162.2, 153.4, 145.2, 112.4, 61.5, 42.2, 42.0, 14.1, 13.93, 13.3 [HRMS $\left(\mathrm{ES},[\mathrm{M}+\mathrm{Na}]^{+}\right)$Found: 238.1047. Calc. for $\mathrm{C}_{10} \mathrm{H}_{17} \mathrm{NO}_{4} \mathrm{Na}$ : 238.1055]; $m / z(\mathrm{CI}) 233\left(12 \%,\left[\mathrm{M}+\mathrm{NH}_{4}\right]^{+}\right), 216(100 \%$, $\left.[\mathrm{M}+\mathrm{H}]^{+}\right), 100(10), 74(13)$.

Reduction to diols and acetonide formation: 3,3-difluoro-2-endo(hydroxymethyl)-7-oxabicyclo[2.2.1] hept-5-en-2-ol 19a

A solution of 12a (12 mmol, 3.7g) in THF $(15 \mathrm{~mL})$ was added to a suspension of lithium aluminium hydride (69 mmol, $2.6 \mathrm{~g})$ in THF $(100 \mathrm{~mL})$ at $0{ }^{\circ} \mathrm{C}$. The grey suspension was refluxed for 3 hours before being cooled to $0^{\circ} \mathrm{C}$ and quenched carefully with water. The white precipitate was dissolved by the cautious addition of concentrated hydrochloric acid. The phases were separated and the aqueous phase extracted with ethyl acetate $(6 \times$ $30 \mathrm{~mL})$. The combined organic extracts were dried $\left(\mathrm{MgSO}_{4}\right)$, filtered and concentrated in vacuo to leave an oil which was purified by column chromatography $(60 \%$ ethyl acetate in light petroleum) to afford diol 19a as cubes (1.4 g, 68\%); mp 104$107^{\circ} \mathrm{C} ; R_{\mathrm{f}}(60 \%$ ethyl acetate in light petroleum) 0.27 (Found: $\mathrm{C}, 47.2 ; \mathrm{H}, 4.6 ; \mathrm{C}_{7} \mathrm{H}_{8} \mathrm{~F}_{2} \mathrm{O}_{3}$ requires: $\mathrm{C}, 47.2 ; \mathrm{H} 4.5 \%$ ); $v_{\max }$ (film)/ $\mathrm{cm}^{-1} 3293 \mathrm{~s}$ br $(\mathrm{OH}), 2918 \mathrm{w}(\mathrm{C}-\mathrm{H}), 1480 \mathrm{~m}(\mathrm{C}-\mathrm{C}), 1310 \mathrm{~s}(\mathrm{C}-\mathrm{O})$, $1278 \mathrm{~s}(\mathrm{C}-\mathrm{O}), 700 \mathrm{w}(\mathrm{HC}=\mathrm{CH}) ; \delta_{\mathrm{H}}\left(300 \mathrm{MHz}, \mathrm{CD}_{3} \mathrm{COCD}_{3}\right)$ 6.73-6.70 $\left(1 \mathrm{H}, \mathrm{m}, \mathrm{H}_{\mathrm{a}} \mathrm{C}=\mathrm{CH}_{\mathrm{b}}\right), 6.50-6.48\left(1 \mathrm{H}, \mathrm{m}, \mathrm{H}_{\mathrm{a}} \mathrm{C}=\mathrm{C} H_{\mathrm{b}}\right)$, $4.86(1 \mathrm{H}, \mathrm{s}, \mathrm{OH}), 4.77-4.75\left(2 \mathrm{H}, \mathrm{m}, \mathrm{CHO}, \mathrm{CHOCF}_{2}\right), 4.18$ $(1 \mathrm{H}, \mathrm{s}, \mathrm{OH}), 3.52\left(1 \mathrm{H}, \mathrm{d},{ }^{2} J 11.0, \mathrm{CH}_{\mathrm{a}} \mathrm{H}_{\mathrm{b}} \mathrm{O}\right), 3.30(1 \mathrm{H}, \mathrm{d}$, $\left.{ }^{2} J 11.0, \mathrm{CH}_{\mathrm{a}} H_{\mathrm{b}} \mathrm{O}\right) ; \delta_{\mathrm{C}}\left(75 \mathrm{MHz}, \mathrm{CD}_{3} \mathrm{COCD}_{3}\right) 138.3\left(\mathrm{t},{ }^{4} J_{\mathrm{C}-\mathrm{F}}\right.$ $1.4), 133.3\left(\mathrm{~d},{ }^{3} J_{\mathrm{C}-\mathrm{F}} 4.5\right), 124.4\left(\mathrm{dd},{ }^{1} J_{\mathrm{C}-\mathrm{F}} 268.4,262.8\right), 87.9(\mathrm{~d}$, $\left.{ }^{3} J_{\mathrm{C}-\mathrm{F}} 5.1\right), 81.6\left(\mathrm{dd},{ }^{2} J_{\mathrm{C}-\mathrm{F}} 29.1,28.0\right), 79.7\left(\mathrm{dd},{ }^{2} J_{\mathrm{C}-\mathrm{F}} 20.4\right.$, $16.4), 64.7\left(\mathrm{~d},{ }^{3} J_{\mathrm{C}-\mathrm{F}} 6.22\right) ; \delta_{\mathrm{F}}\left(282 \mathrm{MHz}, \mathrm{CD}_{3} \mathrm{COCD}_{3}\right)-113.7$ $\left(1 \mathrm{~F}, \mathrm{~d},{ }^{2} J_{\mathrm{C}-\mathrm{F}} 225.7\right),-114.9\left(1 \mathrm{~F}, \mathrm{~d},{ }^{2} J_{\mathrm{C}-\mathrm{F}} 225.7\right)$ [HRMS (CI, $\left[\mathrm{M}+\mathrm{NH}_{4}\right]^{+}$) Found: 196.078525. Calc. for $\mathrm{C}_{7} \mathrm{H}_{8} \mathrm{~F}_{2} \mathrm{O}_{3} \mathrm{NH}_{4}$ 196.077829]; $m / z$ (CI) $196\left(100 \%,\left[\mathrm{M}+\mathrm{NH}_{4}\right]^{+}\right), 68(10)$.

3,3-Difluoro-2-endo-(hydroxymethyl)-7-oxabicyclo[2.2.1]hept-5en-2-ol acetone acetal 20a

A solution of diol 19a $(3.8 \mathrm{mmol}, 0.68 \mathrm{~g})$ in acetone $(100 \mathrm{~mL})$, was added to anhydrous copper sulfate $(1.3 \mathrm{~g})$ and a catalytic amount of para-toluenesulfonic acid monohydrate $(0.19 \mathrm{mmol}$, $0.036 \mathrm{~g}$ ) under a nitrogen atmosphere. The resulting suspension 
was stirred at room temperature for 12 hours. The reaction was quenched by the addition of saturated aqueous ammonium hydroxide $(10 \mathrm{~mL})$ and then extracted with ethyl acetate $(3 \times$ $20 \mathrm{~mL}$ ). The combined organic extracts were washed with $\mathrm{NaHCO}_{3}(2 \times 10 \mathrm{~mL})$ and brine $(10 \mathrm{~mL})$ before being dried $\left(\mathrm{MgSO}_{4}\right)$ and concentrated in vacuo. Flash column chromatography (20\% diethyl ether in light petroleum) afforded acetonide $20 \mathrm{a}$ as rhombi $(0.63 \mathrm{~g}, 77 \%), R_{\mathrm{f}}(20 \%$ ether in light petroleum) 0.16; mp 66-68 ${ }^{\circ} \mathrm{C}$; (Found: $\mathrm{C}, 55.33 ; \mathrm{H}, 5.35 ; \mathrm{C}_{10} \mathrm{H}_{12} \mathrm{~F}_{2} \mathrm{O}_{3}$ requires: $\mathrm{C} 55.05 ; \mathrm{H}, 5.54 \%) ; v_{\max }(\operatorname{mull}) / \mathrm{cm}^{-1} 3106 \mathrm{w}(=\mathrm{C}-\mathrm{H})$, 2991m (C-H), 2939w (C-H), 1483w (C-C), 1454w (C-C), $1166 \mathrm{~s}(\mathrm{C}-\mathrm{O}), 1102 \mathrm{~s}(\mathrm{C}-\mathrm{O}), 728 \mathrm{~m}(\mathrm{HC}=\mathrm{CH}) ; \delta_{\mathrm{H}}(300 \mathrm{MHz}$, $\left.\mathrm{CDCl}_{3}\right) 6.52\left(1 \mathrm{H}, \mathrm{dd}, J 5.9,1.1, H_{\mathrm{a}} \mathrm{C}=\mathrm{CH}_{\mathrm{b}}\right), 6.48(1 \mathrm{H}, \mathrm{d}, J 5.9$, $\left.\mathrm{H}_{\mathrm{a}} \mathrm{C}=\mathrm{CH}_{\mathrm{b}}\right), 4.78-4.75\left(2 \mathrm{H}, \mathrm{m}, \mathrm{CHO}, \mathrm{CHOCF}_{2}\right), 3.95\left(1 \mathrm{H}, \mathrm{d},{ }^{2} \mathrm{~J}\right.$ 9.6, $\left.\mathrm{CH}_{\mathrm{a}} \mathrm{H}_{\mathrm{b}} \mathrm{O}\right), 3.50\left(1 \mathrm{H}, \mathrm{dt},{ }^{2} J 9.6,{ }^{4} J_{\mathrm{H}-\mathrm{F}} 2.1, \mathrm{CH}_{\mathrm{a}} H_{\mathrm{b}} \mathrm{O}\right), 1.44$ $\left(3 \mathrm{H}, \mathrm{s}, \mathrm{C} H_{3}\right), 1.41\left(3 \mathrm{H}, \mathrm{s}, \mathrm{CH}_{3}\right) ; \delta_{\mathrm{C}}\left(75 \mathrm{MHz}, \mathrm{CDCl}_{3}\right) 135.4(\mathrm{t}$, $\left.{ }^{4} J_{\mathrm{C}-\mathrm{F}} 1.4\right), 135.0\left(\mathrm{~d},{ }^{3} J_{\mathrm{C}-\mathrm{F}} 5.1\right), 121.9\left(\mathrm{dd},{ }^{1} J_{\mathrm{C}-\mathrm{F}} 271.8,264.5\right)$, $111.9,85.9\left(\mathrm{~d},{ }^{3} J_{\mathrm{C}-\mathrm{F}} 5.6\right), 83.9\left(\mathrm{dd},{ }^{2} J_{\mathrm{C}-\mathrm{F}} 22.6,15.3\right), 80.5(\mathrm{dd}$, $\left.{ }^{2} J_{\mathrm{C}-\mathrm{F}} 29.1,27.4\right), 66.4\left(\mathrm{~d},{ }^{3} J_{\mathrm{C}-\mathrm{F}} 6.2\right), 25.8,25.5 ; \delta_{\mathrm{F}}(282 \mathrm{MHz}$, $\left.\mathrm{CDCl}_{3}\right)-108.6\left(1 \mathrm{~F}, \mathrm{~d},{ }^{3} J_{\mathrm{F}-\mathrm{F}} 221.3\right),-111.7\left(1 \mathrm{~F}, \mathrm{~d},{ }^{3} J_{\mathrm{F}-\mathrm{F}} 221.3\right)$ [HRMS (ES, $\left[\mathrm{M}+\mathrm{Na}^{+}\right.$) Found: 241.0660. Calc. for $\left.\mathrm{C}_{10} \mathrm{H}_{12} \mathrm{~F}_{2} \mathrm{O}_{3} \mathrm{Na} 241.0652\right] ; m / z(\mathrm{CI}) 236\left(15 \%\left[\mathrm{M}+\mathrm{NH}_{4}\right]^{+}\right), 219$ $\left(100 \%[\mathrm{M}+\mathrm{H}]^{+}\right)$.

\section{3,3-Difluoro-2-endo-(hydroxymethyl)-1-methyl-7-oxabicyclo- [2.2.1]hept-5-en-2-ol 21 a and 3,3-difluoro-2-exo-(hydroxymethyl)- 1-methyl-7-oxabicyclo[2.2.1] hept-5-en-2-ol 21b}

Diols 21a and 21b were prepared as for 19a from adducts 13a and 13b (16 mmol, $5.51 \mathrm{~g})$, and lithium aluminium hydride (96 mmol, $4.6 \mathrm{~g})$ in THF $(160 \mathrm{~mL})$. The resulting orange oil was purified by column chromatography $(50 \%$ diethyl ether in light petroleum) to afford an inseparable mixture of the diols 21a and $21 \mathrm{~b}\left(2.5 \mathrm{~g}, 80 \%, 1: 1,98 \%\right.$ by GC) as an oil $R_{\mathrm{f}}(50 \%$ diethyl ether in light petroleum) $0.19 ; v_{\max }$ (film) $/ \mathrm{cm}^{-1} 3440 \mathrm{~s}$ br $(\mathrm{OH})$, 2983m (C-H), 2940m (C-H), 1686w (C-C), 1449w (C-C), $1299 \mathrm{~s}(\mathrm{C}-\mathrm{O}), 1165 \mathrm{~s}(\mathrm{C}-\mathrm{O}), 712 \mathrm{~m}(\mathrm{HC}=\mathrm{CH}) ; \delta_{\mathrm{H}}(300 \mathrm{MHz}$, $\left.\mathrm{CDCl}_{3}\right) 6.44-6.38(4 \mathrm{H}, \mathrm{m}, \mathrm{HC}=\mathrm{CH}), 4.70\left(1 \mathrm{H}, \mathrm{d},{ }^{3} J_{\mathrm{H}-\mathrm{F}} 7.0\right.$, $\left.\mathrm{CHOCF}_{2}\right), 4.64\left(1 \mathrm{H}, \mathrm{d}, J\right.$ 2.6, $\left.\mathrm{CHOCF}_{2}\right), 4.00-3.32$ (5H, env., $\left.\mathrm{CH}_{2} \mathrm{OH}, \mathrm{OH}\right), 2.81(1 \mathrm{H}$, br. s, $\mathrm{OH}), 1.53\left(3 \mathrm{H}, \mathrm{s}, \mathrm{CH}_{3}\right), 1.46$ $\left(3 \mathrm{H}, \mathrm{s}, \mathrm{CH}_{3}\right) ; \delta_{\mathrm{C}}\left(75 \mathrm{MHz}, \mathrm{CDCl}_{3}\right) 141.6\left(\mathrm{t},{ }^{4} J_{\mathrm{C}-\mathrm{F}} 1.7\right), 140.3(\mathrm{t}$, $\left.{ }^{4} J_{\mathrm{C}-\mathrm{F}} 1.4\right), 132.7\left(\mathrm{~d},{ }^{3} J_{\mathrm{C}-\mathrm{F}} 3.4\right), 131.4\left(\mathrm{~d},{ }^{3} J_{\mathrm{C}-\mathrm{F}} 4.5\right), 124.7(\mathrm{t}$, $\left.{ }^{1} J_{\mathrm{C}-\mathrm{F}} 266.2\right), 123.7\left(\mathrm{t},{ }^{1} J_{\mathrm{C}-\mathrm{F}} 266.8\right), 92.2\left(\mathrm{dd},{ }^{3} J_{\mathrm{C}-\mathrm{F}} 4.0,1.1\right), 89.4$ $\left(\mathrm{t},{ }^{3} J_{\mathrm{C}-\mathrm{F}} 2.3\right), 81.0\left(\mathrm{t},{ }^{2} J_{\mathrm{C}-\mathrm{F}} 28.0\right), 80.2\left(\mathrm{t},{ }^{2} J_{\mathrm{C}-\mathrm{F}} 28.3\right), 78.8(\mathrm{dd}$, $\left.{ }^{2} J_{\mathrm{C}-\mathrm{F}} 19.2,17.0\right), 77.4\left(\mathrm{dd},{ }^{2} J_{\mathrm{C}-\mathrm{F}} 19.8,17.0\right), 64.13\left(\mathrm{dd},{ }^{3} J_{\mathrm{C}-\mathrm{F}} 4.5\right.$, $1.7), 62.7\left(\mathrm{~d},{ }^{3} J_{\mathrm{C}-\mathrm{F}} 11.8\right), 14.1,14.0 ; \delta_{\mathrm{F}}\left(282 \mathrm{MHz}, \mathrm{CDCl}_{3}\right)$ $-107.9\left(1 \mathrm{~F}, \mathrm{~d},{ }^{2} J_{\mathrm{F}-\mathrm{F}} 224.1\right),-113.1\left(1 \mathrm{~F}, \mathrm{~d}^{2} J_{\mathrm{F}-\mathrm{F}} 228.3\right),-113.7$ $\left(1 \mathrm{~F}, \mathrm{~d}^{2} J_{\mathrm{F}-\mathrm{F}} 228.3\right),-115.5\left(1 \mathrm{~F}, \mathrm{dd},{ }^{2} J_{\mathrm{F}-\mathrm{F}} 224.1,{ }^{3} J_{\mathrm{F}-\mathrm{H}} 7.0\right)$ [HRMS (ES, $[\mathrm{M}+\mathrm{Na}]^{+}$) Found: 215.0501. Calc. for $\mathrm{C}_{8} \mathrm{H}_{10^{-}}$ $\left.\mathrm{F}_{2} \mathrm{O}_{5} \mathrm{Na} 215.0496\right] ; \mathrm{m} / z$ (ES) $215\left(100 \%[\mathrm{M}+\mathrm{Na}]^{+}\right)$. Distinct endo and exo signals cannot be assigned because of overlap.

\section{3,3-Difluoro-2-endo-(hydroxymethyl)-1-methyl-7-oxabicyclo- [2.2.1]hept-5-en-2-ol acetone acetal 22a and 3,3-difluoro-2-exo- (hydroxymethyl)-1-methyl-7-oxabicyclo[2.2.1] hept-5-en-2-ol acetone acetal 22b}

The acetonides were prepared as for 20a from the mixture of diols $21 \mathrm{a}$ and $21 \mathrm{~b}(0.58 \mathrm{~g}, 3.0 \mathrm{mmol})$, acetone $(100 \mathrm{~mL})$, copper sulfate $(6 \mathrm{mmol}, 1.2 \mathrm{~g})$ and a catalytic amount of para-toluenesulfonic acid monohydrate $(0.15 \mathrm{mmol}, 0.029 \mathrm{~g})$. The resulting brown oil was purified by column chromatography (10\% diethyl ether in light petroleum) to afford endo-acetonide 22a $(0.79 \mathrm{~g}$, $34 \%, 94 \%$ by GC) as needles; mp $129-131{ }^{\circ} \mathrm{C} ; R_{\mathrm{f}}(10 \%$ diethyl ether in light petroleum) $0.47 ; v_{\max }$ (film) $/ \mathrm{cm}^{-1} 2989 \mathrm{~m}(\mathrm{C}-\mathrm{H})$, 2936m (C-H), 1489w (C-C), 1455w (C-C), 1299m (C-O), $1210 \mathrm{~s}(\mathrm{C}-\mathrm{O}), 1127 \mathrm{~s}(\mathrm{C}-\mathrm{O}), 1085 \mathrm{~s}(\mathrm{C}-\mathrm{O}), 723 \mathrm{~m}(\mathrm{HC}=\mathrm{CH})$; $\delta_{\mathrm{H}}\left(300 \mathrm{MHz}, \mathrm{CDCl}_{3}\right) 6.46\left(1 \mathrm{H}, \mathrm{d}, J 5.9, H_{\mathrm{a}} \mathrm{C}=\mathrm{CH}_{\mathrm{b}}\right), 6.30(1 \mathrm{H}$, $\left.\mathrm{d}, J 5.9, \mathrm{H}_{\mathrm{a}} \mathrm{C}=\mathrm{C} H_{\mathrm{b}}\right), 4.74-4.72\left(1 \mathrm{H}, \mathrm{m}, \mathrm{CHOCF}_{2}\right), 4.00(1 \mathrm{H}, \mathrm{d}$, ${ }^{2} J$ 9.6, $\left.\mathrm{CH}_{\mathrm{a}} \mathrm{H}_{\mathrm{b}} \mathrm{O}\right), 3.42-3.39\left(1 \mathrm{H}, \mathrm{m}, \mathrm{CH}_{\mathrm{a}} H_{\mathrm{b}} \mathrm{O}\right), 1.53(3 \mathrm{H}, \mathrm{s}$, $\left.\mathrm{C}\left(\mathrm{CH}_{3}\right) \mathrm{O}\right), 1.47\left(3 \mathrm{H}, \mathrm{s}, \mathrm{CH}_{3}\right), 1.43\left(3 \mathrm{H}, \mathrm{s}, \mathrm{CH}_{3}\right) ; \delta_{\mathrm{C}}(75 \mathrm{MHz}$,
$\left.\mathrm{CDCl}_{3}\right) 139.2\left(\mathrm{t},{ }^{4} J_{\mathrm{C}-\mathrm{F}} 1.4\right), 135.2\left(\mathrm{~d},{ }^{3} J_{\mathrm{C}-\mathrm{F}} 5.1\right), 122.7\left(\mathrm{dd},{ }^{1} J_{\mathrm{C}-\mathrm{F}}\right.$ 271.0, 264.2), 111.8, $90.1\left(\mathrm{~d},{ }^{3} J_{\mathrm{C}-\mathrm{F}} 5.1\right), 84.2\left(\mathrm{dd},{ }^{2} J_{\mathrm{C}-\mathrm{F}} 21.5\right.$, 15.3), $20.3\left(\mathrm{dd},{ }^{2} J_{\mathrm{C}-\mathrm{F}} 28.8,27.1\right), 66.6\left(\mathrm{~d},{ }^{3} J_{\mathrm{C}-\mathrm{F}} 6.2\right), 25.7,25.4$, $13.2 ; \delta_{\mathrm{F}}\left(282 \mathrm{MHz}, \mathrm{CDCl}_{3}\right)-107.5\left(1 \mathrm{~F}, \mathrm{~d},{ }^{2} J_{\mathrm{F}-\mathrm{F}} 219.7\right),-109.9$ $\left(1 \mathrm{~F}, \mathrm{dd},{ }^{2} J_{\mathrm{F}-\mathrm{F}} 219.7,{ }^{3} J_{\mathrm{F}-\mathrm{H}} 4.5\right)$; [HRMS (ES, $\left.[\mathrm{M}+\mathrm{Na}]^{+}\right)$Found 255.0810. Calc. for $\mathrm{C}_{11} \mathrm{H}_{14} \mathrm{O}_{3} \mathrm{~F}_{2} \mathrm{Na}$ 255.0809]; $\mathrm{m} / \mathrm{z}$ (ES) 255 $\left(100 \%[\mathrm{M}+\mathrm{Na}]^{+}\right)$: and exo-acetonide $22 \mathrm{~b}(0.83 \mathrm{~g}, 37 \%, 100 \%$ by GC) as needles mp $120-123^{\circ} \mathrm{C} ; R_{\mathrm{f}}(10 \%$ diethyl ether in light petroleum) 0.22 ; $v_{\max }(\mathrm{film}) / \mathrm{cm}^{-1} 3081 \mathrm{w}(\mathrm{C}-\mathrm{H}), 2990 \mathrm{w}(\mathrm{C}-\mathrm{H})$, 1686w $(\mathrm{C}=\mathrm{C}), 1451 \mathrm{w}(\mathrm{C}-\mathrm{C}), 1228 \mathrm{w}(\mathrm{C}-\mathrm{O}), 1089 \mathrm{w}(\mathrm{C}-\mathrm{O}), 702 \mathrm{w}$ $(\mathrm{HC}=\mathrm{CH}) ; \delta_{\mathrm{H}}\left(300 \mathrm{MHz}, \mathrm{CDCl}_{3}\right) 6.46-6.45(2 \mathrm{H}, \mathrm{m}, \mathrm{HC}=\mathrm{CH})$, $4.74\left(1 \mathrm{H}, \mathrm{d},{ }^{3} J_{\mathrm{H}-\mathrm{F}} 7.3, \mathrm{CHOCF}_{2}\right), 4.34\left(1 \mathrm{H}, \mathrm{d},{ }^{2} J 10.3, \mathrm{CH}_{\mathrm{a}-}\right.$ $\left.\mathrm{H}_{\mathrm{b}} \mathrm{O}\right), 4.09\left(1 \mathrm{H}, \mathrm{d},{ }^{2} J 10.3, \mathrm{CH}_{\mathrm{a}} \mathrm{H}_{\mathrm{b}} \mathrm{O}\right), 1.53\left(3 \mathrm{H}, \mathrm{s}, \mathrm{C}\left(\mathrm{CH}_{3}\right) \mathrm{O}\right)$, $1.46\left(3 \mathrm{H}, \mathrm{s}, \mathrm{CH}_{3}\right), 1.37\left(3 \mathrm{H}, \mathrm{s}, \mathrm{CH}_{3}\right) ; \delta_{\mathrm{C}}\left(75 \mathrm{MHz}, \mathrm{CDCl}_{3}\right) 141.7$ $\left(\mathrm{t},{ }^{4} J_{\mathrm{C}-\mathrm{F}} 1.4\right), 131.6\left(\mathrm{~d},{ }^{3} J_{\mathrm{C}-\mathrm{F}} 5.1\right), 123.3\left(\mathrm{dd},{ }^{1} J_{\mathrm{C}-\mathrm{F}} 267.3,266.2\right)$, $111.1,88.5\left(\mathrm{t},{ }^{3} J_{\mathrm{C}-\mathrm{F}} 1.7\right), 84.13\left(\mathrm{dd},{ }^{2} J_{\mathrm{C}-\mathrm{F}} 21.2,16.7\right), 81.22(\mathrm{dd}$, $\left.{ }^{2} J_{\mathrm{C}-\mathrm{F}} 28.3,27.1\right), 65.0,26.8,24.4,13.7 ; \delta_{\mathrm{F}}\left(282 \mathrm{MHz}, \mathrm{CDCl}_{3}\right)$ $-107.8\left(1 \mathrm{~F}, \mathrm{~d},{ }^{2} J_{\mathrm{F}-\mathrm{F}} 223.8\right),-110.3\left(1 \mathrm{~F}, \mathrm{dd},{ }^{2} J_{\mathrm{F}-\mathrm{F}} 223.8,{ }^{3} J_{\mathrm{F}-\mathrm{H}}\right.$ 7.3) [HRMS (ES, $[\mathrm{M}+\mathrm{Na}]^{+}$) Found: 255.0799. Calc. for $\left.\mathrm{C}_{11} \mathrm{H}_{14} \mathrm{~F}_{2} \mathrm{O}_{5} \mathrm{Na} 255.0809\right]$; $m / z$ (ES) $255\left(100 \%[\mathrm{M}+\mathrm{Na}]^{+}\right)$. Satisfactory microanalyses could not be obtained for these compounds.

\section{3,3-Difluoro-2-exo-(hydroxymethyl)-1,6-dimethyl-7-oxa- bicyclo[2.2.1] hept-5-en-2-ol 23b}

Diol 23b was prepared from exo cycloadduct 14b $(9.8 \mathrm{mmol}$, $3.4 \mathrm{~g})$ and lithium aluminum hydride $(57.6 \mathrm{mmol}, 2.76 \mathrm{~g})$ in THF $(106 \mathrm{~mL})$ at reflux for 6 hours. Usual work-up afforded a yellow solid, which was washed with hexane to yield diol 23b as fine needles $(2.16 \mathrm{~g}, 89 \%)$; mp $87-89^{\circ} \mathrm{C} ; R_{\mathrm{f}}(60 \%$ diethyl ether in light petroleum) 0.26 (Found: C, 52.67; H, 5.86; $\mathrm{C}_{9} \mathrm{H}_{12} \mathrm{~F}_{2} \mathrm{O}_{3}$ requires: $\mathrm{C}, 52.43 ; \mathrm{H}, 5.87 \%) ; \delta_{\mathrm{H}}\left(250 \mathrm{MHz}, \mathrm{CDCl}_{3}\right) 6.0(1 \mathrm{H}, \mathrm{s},=\mathrm{CH}), 4.58$ $\left(1 \mathrm{H}, \mathrm{d},{ }^{3} \mathrm{~J}_{\mathrm{H}-\mathrm{F}} 6.8, \mathrm{CHOCF}{ }_{2}\right), 4.03\left(1 \mathrm{H}, \mathrm{dd},{ }^{2} J 11.7,{ }^{4} \mathrm{~J}_{\mathrm{H}-\mathrm{F}} 1.6\right.$, $\left.\mathrm{C}_{\mathrm{a}} \mathrm{H}_{\mathrm{b}} \mathrm{O}\right), 3.76\left(1 \mathrm{H}, \mathrm{d},{ }^{2} J 11.7, \mathrm{CH}_{\mathrm{a}} \mathrm{H}_{\mathrm{b}} \mathrm{O}\right), 3.08(1 \mathrm{H}, \mathrm{d}, J 2.5$, $\mathrm{OH}), 2.18(1 \mathrm{H}$, br. s, $\mathrm{OH}), 1.91\left(3 \mathrm{H}, \mathrm{s},=\mathrm{CCH}_{3}\right), 1.44(3 \mathrm{H}, \mathrm{s}$, $\left.\mathrm{C}\left(\mathrm{CH}_{3}\right) \mathrm{O}\right) ; \delta_{\mathrm{C}}\left(75 \mathrm{MHz}, \mathrm{CD}_{3} \mathrm{OD}\right) 153.6,126.3\left(\mathrm{t},{ }^{1} J_{\mathrm{C}-\mathrm{F}} 264.8\right)$, $125.4\left(\mathrm{~d},{ }^{3} J_{\mathrm{C}-\mathrm{F}} 6.0\right), 92.4,81.5\left(\mathrm{t}^{2} J_{\mathrm{C}-\mathrm{F}} 27.9\right), 79.2\left(\mathrm{dd},{ }^{2} J_{\mathrm{C}-\mathrm{F}} 16.8\right.$, 20.4), $63.6\left(\mathrm{~d},{ }^{2} J_{\mathrm{C}-\mathrm{F}} 13.4\right), 14.4,13.9 ; \delta_{\mathrm{F}}\left(235 \mathrm{MHz}, \mathrm{CDCl}_{3}\right)$ $-108.8\left(1 \mathrm{~F}, \mathrm{~d},{ }^{2} J_{\mathrm{F}-\mathrm{F}} 222.9\right),-117.0\left(1 \mathrm{~F}, \mathrm{dd},{ }^{2} J_{\mathrm{F}-\mathrm{F}} 222.9,{ }^{3} J_{\mathrm{F}-\mathrm{H}} 6.8\right)$; $\mathrm{m} / \mathrm{z}$ (ES) 205 (10\%, M-H), 185 (19), 165 (100), 149 (22), 137 (76).

\section{3,3-Difluoro-2-exo-(hydroxymethyl)-1,6-dimethyl-7-oxa- bicyclo[2.2.1] hept-5-en-2-ol acetone acetal 24b}

The acetonide was prepared from a mixture of diol 23b $(4.8 \mathrm{mmol}, 1.0 \mathrm{~g})$, anhydrous $\mathrm{CuSO}_{4}(15 \mathrm{mmol}, 2.4 \mathrm{~g})$ and p-toluenesulfonic acid monohydrate $(0.24 \mathrm{mmol}, 0.046 \mathrm{~g})$ in acetone $(150 \mathrm{~mL})$ at room temperature over 18 hours. Usual work-up afforded a light brown semi-solid, which was triturated with hexane to afford exo-acetal $\mathbf{2 4 b}$ as cubes $(1.1 \mathrm{~g}, 91 \%) ; R_{\mathrm{f}}$ ( $20 \%$ diethyl ether in light petroleum) $0.11 ; \mathrm{mp} 55-57^{\circ} \mathrm{C}$; (Found: $\mathrm{C}, 58.71 ; \mathrm{H}, 6.70 ; \mathrm{C}_{12} \mathrm{H}_{16} \mathrm{~F}_{2} \mathrm{O}_{3}$ requires: $\mathrm{C}, 58.53 ; \mathrm{H}$, $6.55 \%) ; \delta_{\mathrm{H}}\left(250 \mathrm{MHz}, \mathrm{CDCl}_{3}\right) 6.0(1 \mathrm{H}, \mathrm{s},=\mathrm{CH}), 4.59(1 \mathrm{H}, \mathrm{d}$, $\left.{ }^{3} J_{\mathrm{H}-\mathrm{F}} 6.1, \mathrm{CHOCF}_{2}\right), 4.32\left(1 \mathrm{H}, \mathrm{d},{ }^{2} J 10.3, \mathrm{CH}_{\mathrm{a}} \mathrm{H}_{\mathrm{b}} \mathrm{O}\right), 4.10(1 \mathrm{H}$, $\left.\mathrm{d},{ }^{2} \mathrm{~J} 10.3, \mathrm{CH}_{\mathrm{a}} \mathrm{H}_{\mathrm{b}} \mathrm{O}\right), 1.91\left(3 \mathrm{H}, \mathrm{s},=\mathrm{CCH}_{3}\right), 1.47(3 \mathrm{H}, \mathrm{s}$, $\left.\mathrm{C}\left(\mathrm{CH}_{3}\right) \mathrm{O}\right), 1.46\left(3 \mathrm{H}, \mathrm{s}, \mathrm{CH}_{3}\right), 1.37\left(3 \mathrm{H}, \mathrm{s}, \mathrm{CH}_{3}\right) ; \delta_{\mathrm{C}}(75 \mathrm{MHz}$, $\left.\mathrm{CDCl}_{3}\right) 151.8,124.7\left(\mathrm{~d},{ }^{3} J_{\mathrm{C}-\mathrm{F}} 4.8\right), 123.4\left(\mathrm{t},{ }^{1} J_{\mathrm{C}-\mathrm{F}} 266.2\right), 111.2$, $89.6,84.8\left(\mathrm{dd},{ }^{2} J_{\mathrm{C}-\mathrm{F}} 21.1,16.3\right), 80.1\left(\mathrm{t},{ }^{2} J_{\mathrm{C}-\mathrm{F}} 27.7\right), 65.3,26.9$, $24.7,14.4,12.5 ; \delta_{\mathrm{F}}\left(235 \mathrm{MHz}, \mathrm{CDCl}_{3}\right)-109.2\left(1 \mathrm{~F}, \mathrm{~d},{ }^{2} J_{\mathrm{F}-\mathrm{F}}\right.$ 222.6), $-111.7\left(1 \mathrm{~F}, \mathrm{dd},{ }^{2} J_{\mathrm{F}-\mathrm{F}} 222.6,{ }^{3} \mathrm{~J}_{\mathrm{F}-\mathrm{H}} 6.1\right)$ [HRMS (EI) Found: 246.10675. Calc. for $\mathrm{C}_{12} \mathrm{H}_{16} \mathrm{~F}_{2} \mathrm{O}_{3}$ 246.2498.]; $\mathrm{m} / \mathrm{z}$ (EI) $246(40 \%), 231(100)$.

Hydrostannylation reactions: 3,3-difluoro-2 $S^{*}$-(hydroxymethyl)$6 S^{*}$-tributylstannyl-7-oxa-1 $R^{*}, 4 S^{*}$-bicyclo[2.2.1] heptan-ol dimethyl acetonide 25 and 3,3-Difluoro- $2 S^{*}$-(hydroxymethyl)-5 $5 S^{*}$ tributylstannyl-7-oxa-1 $R^{*}, 4 S^{*}$-bicyclo[2.2.1] heptan-2-ol dimethyl acetonide 26

Freshly-distilled tributyltin hydride $(6.7 \mathrm{mmol}, 1.8 \mathrm{~mL})$ in toluene $(10 \mathrm{~mL})$ was added over one hour to a solution of acetonide 
20a (3.2 mmol, $0.7 \mathrm{~g}), \mathrm{Pd}_{2} \mathrm{dba}_{3} \cdot \mathrm{CHCl}_{3}(0.07 \mathrm{mmol}, 0.07 \mathrm{~g})$ and triphenylphosphine $(0.31 \mathrm{mmol}, 0.08 \mathrm{~g})$ in toluene (40 $\mathrm{mL}$ ). The resulting yellow solution was stirred at room temperature for 16 hours. The solvent was removed in vacuo and the resulting oil purified by column chromatography to afford (in order of elution) stannane $26(0.30 \mathrm{~g}, 19 \%) ; R_{\mathrm{f}}(5 \% \mathrm{di}-$ ethyl ether in light petroleum) $0.26 ; v_{\max }\left(\right.$ film) $/ \mathrm{cm}^{-1} 2928 \mathrm{~s} \mathrm{br}$ $(\mathrm{C}-\mathrm{H}), 1455 \mathrm{~m}(\mathrm{C}-\mathrm{C}), 1097 \mathrm{~s}(\mathrm{C}-\mathrm{O}) ; \delta_{\mathrm{H}}\left(400 \mathrm{MHz}, \mathrm{CDCl}_{3}\right)$ $4.48-4.46(1 \mathrm{H}, \mathrm{m}, \mathrm{CHO}), 4.27\left(1 \mathrm{H}, \mathrm{d},{ }^{3} J_{\mathrm{H}-\mathrm{Sn}} 15.6,{ }^{3} J_{\mathrm{H}-\mathrm{F}} 7.2\right.$, $\left.\mathrm{CHOCF}_{2}\right), 4.22\left(1 \mathrm{H}, \mathrm{d},{ }^{2} J 9.4, \mathrm{CH}_{\mathrm{a}} \mathrm{H}_{\mathrm{b}} \mathrm{O}\right), 3.85\left(1 \mathrm{H}, \mathrm{dt},{ }^{2} J 9.4\right.$, $\left.{ }^{4} J_{\mathrm{H}-\mathrm{F}} 2.8, \mathrm{CH}_{\mathrm{a}} H_{\mathrm{b}} \mathrm{O}\right), 1.95-1.55\left(3 \mathrm{H}, \mathrm{m}, \mathrm{CH}_{2} \mathrm{CHSn}, \mathrm{CHSn}\right)$, 1.54-1.39 (12H, env., $\left.\mathrm{SnCH}_{2} \mathrm{CH}_{2} \mathrm{CH}_{2}, \mathrm{C}\left(\mathrm{CH}_{3}\right)_{2}\right), 1.37-1.28$ $\left(6 \mathrm{H}, \mathrm{SnCH}_{2} \mathrm{CH}_{2}\right), 1.09-0.73\left(15 \mathrm{H}, \mathrm{SnCH}_{2}, \mathrm{CH}_{2} \mathrm{CH}_{3}\right) ; \delta_{\mathrm{C}}(100$ $\left.\mathrm{MHz}, \mathrm{CDCl}_{3}\right) 121.9$ (dd, $\left.{ }^{1} J_{\mathrm{C}-\mathrm{F}} 272.0,263.0\right), 111.7,87.4$ $\left(\mathrm{dd},{ }^{2} J_{\mathrm{C}-\mathrm{F}} 26.8,16.0\right), 84.6\left(\mathrm{~d},{ }^{3} J_{\mathrm{C}-\mathrm{F}} 5.7\right), 82.7\left(\mathrm{dd},{ }^{2} J_{\mathrm{C}-\mathrm{F}}\right.$ 28.2, 25.4), $64.9\left(\mathrm{~d},{ }^{3} J_{\mathrm{C}-\mathrm{F}} 7.5\right), 29.0\left(\mathrm{t},{ }^{3} J_{\mathrm{C}-\mathrm{Sn}} 10.2\right), 28.5,27.4(\mathrm{t}$, $\left.{ }^{2} J_{\mathrm{C}-\mathrm{Sn}} 27.3\right), 25.9,25.8,16.9\left(\mathrm{t},{ }^{3} J_{\mathrm{C}-\mathrm{Sn}} 150.1\right), 13.6,8.8\left(\mathrm{t},{ }^{3} J_{\mathrm{C}-\mathrm{Sn}}\right.$ $157.6) ; \delta_{\mathrm{F}}\left(282 \mathrm{MHz}, \mathrm{CDCl}_{3}\right)-110.6\left(\mathrm{dd},{ }^{2} J_{\mathrm{F}-\mathrm{F}} 223.0,{ }^{3} J_{\mathrm{F}-\mathrm{H}}\right.$ 7.2), -120.7 (d, ${ }^{2} J_{\mathrm{F}-\mathrm{F}}$ 223.0); [HRMS (EI, [M]+) Found 506.19607. Calc. for $\left.\mathrm{C}_{22} \mathrm{H}_{40} \mathrm{O}_{3} \mathrm{~F}_{2}{ }^{116} \mathrm{Sn} 506.19665\right]$; $\mathrm{m} / \mathrm{z}$ (EI) 506 (7\%), 281 (18), 251 (100), 199 (98), 171 (52), 141 (86), 121 (52): and stannane 25 as a colourless oil $(0.59 \mathrm{~g}, 38 \%) R_{\mathrm{f}}(5 \%$ diethyl ether in light petroleum) $0.18 ; v_{\max }(\mathrm{film}) / \mathrm{cm}^{-1} 2928 \mathrm{~s}$ br $(\mathrm{C}-\mathrm{H}), 1455 \mathrm{~m}(\mathrm{C}-\mathrm{C}), 1097 \mathrm{~s}(\mathrm{C}-\mathrm{O}) ; \delta_{\mathrm{H}}\left(300 \mathrm{MHz}, \mathrm{CDCl}_{3}\right)$ 4.48-4.44 (1H, m, CHO $), 4.23\left(1 \mathrm{H}, \mathrm{d}, J 3.5, \mathrm{CHOCF}_{2}\right), 4.16$ $\left(1 \mathrm{H}, \mathrm{d},{ }^{2} J 9.4, \mathrm{CH}_{\mathrm{a}} \mathrm{H}_{\mathrm{b}} \mathrm{O}\right), 3.72\left(1 \mathrm{H}, \mathrm{dt},{ }^{2} J 9.4,{ }^{4} J_{\mathrm{H}-\mathrm{F}} 2.6\right.$, $\left.\mathrm{CH}_{\mathrm{a}} \mathrm{H}_{\mathrm{b}} \mathrm{O}\right), 2.13-2.05(1 \mathrm{H}, \mathrm{m}, \mathrm{CHSn}), 1.70-0.72(35 \mathrm{H}$, env., all $n$-Bu, $\left.\mathrm{CHCH}_{2}, \mathrm{CH}_{3}\right) ; \delta_{\mathrm{C}}\left(75 \mathrm{MHz}, \mathrm{CDCl}_{3}\right) 124.7$ (t, $\left.{ }^{1} J_{\mathrm{C}-\mathrm{F}} 268.5\right), 112.3,89.3\left(\mathrm{dd},{ }^{2} J_{\mathrm{C}-\mathrm{F}} 25.9,16.3\right), 87.5\left(\mathrm{~d},{ }^{3} J_{\mathrm{C}-\mathrm{F}} 5.4\right)$, $81.0\left(\mathrm{t},{ }^{2} J_{\mathrm{C}-\mathrm{F}} 27.1\right), 65.3\left(\mathrm{~d},{ }^{3} J_{\mathrm{C}-\mathrm{F}} 8.4\right), 29.4\left(\mathrm{t},{ }^{3} J_{\mathrm{C}-\mathrm{Sn}} 10.2\right)$, $27.8\left(\mathrm{t},{ }^{2} J_{\mathrm{C}-\mathrm{Sn}} 27.1\right), 26.3,26.0,18.8\left(\mathrm{t},{ }^{1} J_{\mathrm{C}-\mathrm{Sn}} 141.9\right), 14.1$, $9.2\left(\mathrm{t},{ }^{1} J_{\mathrm{C}-\mathrm{Sn}} 157.9\right) ; \delta_{\mathrm{F}}\left(282 \mathrm{MHz}, \mathrm{CDCl}_{3}\right)-113.1(\mathrm{dd}$, $\left.{ }^{2} J_{\mathrm{F}-\mathrm{F}} 226.6, \quad 5.5\right), \quad-119.7\left(\mathrm{~d},{ }^{2} J_{\mathrm{F}-\mathrm{F}} 226.6\right)$ [HRMS (EI, $[\mathrm{M}]^{+}$) Found 506.19667. Calc. for $\mathrm{C}_{22} \mathrm{H}_{40} \mathrm{O}_{3} \mathrm{~F}_{2}{ }^{116} \mathrm{Sn}$ 506.19665]; $\mathrm{m} / z$ (EI) 506 (12\%), 453 (39), 395 (35), 291 (53), 181 (100). Both stannane products were contaminated with traces of trialkyltin compounds which could not be removed by chromatography.

\section{3,3-Difluoro-2 $S^{*}$-(hydroxymethyl)-1-methyl-5 $R^{*}$-tributyl- stannyl-7-oxa-1 $S^{*}, 4 S^{*}$-bicyclo[2.2.1]heptan-2-ol dimethyl acetonide 27}

Prepared as for $\mathbf{2 5}$ and $\mathbf{2 6}$ from acetonide 22a ( $2 \mathrm{mmol}, 0.46 \mathrm{~g})$, tributyltin hydride $(4.2 \mathrm{mmol}, 1.2 \mathrm{~mL}), \mathrm{Pd}_{2} \mathrm{dba}_{3} \cdot \mathrm{CHCl}_{3}(0.04$ mmol, $0.04 \mathrm{~g})$, triphenylphosphine $(0.2 \mathrm{mmol}, 0.04 \mathrm{~g})$ and toluene $(30 \mathrm{~mL})$. After stirring at room temperature for 16 hours the solvent was removed in vacuo and the resulting oil purified by column chromatography $(5 \%$ diethyl ether in light petroleum) to afford stannane $27(0.51 \mathrm{~g}, 50 \%)$ as a colourless oil; $R_{\mathrm{f}}$ ( $5 \%$ diethyl ether in light petroleum) $0.47 ; v_{\max }($ film $) / \mathrm{cm}^{-1} 2957 \mathrm{~s}$ (C-H), 2928s (C-H), 2872s (C-H), 2854s (C-H), $1461 \mathrm{~m}(\mathrm{C}-\mathrm{C})$, $1098 \mathrm{~s}(\mathrm{C}-\mathrm{O}) ; \delta_{\mathrm{H}}\left(300 \mathrm{MHz}, \mathrm{CDCl}_{3}\right) 4.23-4.08(2 \mathrm{H}, \mathrm{m}$, [including $\left.\left.4.17\left(1 \mathrm{H}, \mathrm{d},{ }^{2} J 10.2, \mathrm{CH}_{\mathrm{a}} \mathrm{H}_{\mathrm{b}} \mathrm{O}\right)\right], \mathrm{CHOCF}_{2}\right), 3.99(1 \mathrm{H}, \mathrm{d}$, $\left.{ }^{2} J 10.2, \mathrm{CH}_{\mathrm{a}} H_{\mathrm{b}} \mathrm{O}\right), 2.58-2.38\left(1 \mathrm{H}, \mathrm{m}, \mathrm{CH}_{\mathrm{a}} \mathrm{H}_{\mathrm{b}} \mathrm{CHSn}\right), 1.78-1.72$ $(1 \mathrm{H}, \mathrm{m}, \mathrm{CHSn}), 1.56-1.19\left(26 \mathrm{H}, \mathrm{CH}_{\mathrm{a}} \mathrm{H}_{\mathrm{b}} \mathrm{CHSn}, \mathrm{SnCH}_{2} \mathrm{CH}_{2}\right.$, $\left.\mathrm{SnCH}_{2} \mathrm{CH}_{2} \mathrm{CH}_{2}, \mathrm{C}\left(\mathrm{CH}_{3}\right)_{2}, \mathrm{C}\left(\mathrm{CH}_{3}\right) \mathrm{O}\right), 0.91-0.85(15 \mathrm{H}, \mathrm{m}$, $\left.\left.\mathrm{CH}_{2} \mathrm{Sn}, \mathrm{CH}_{2} \mathrm{CH}_{3}\right){ }^{3} J_{\mathrm{H}-\mathrm{F}} 9.0\right), 4.23(1 \mathrm{H}, \mathrm{d}, J 1.9), 4.06(1 \mathrm{H}, \mathrm{d}$, $J 10.1), 2.54$ (1H, dd, $J 10.1), 1.84-1.75(1 \mathrm{H}, \mathrm{m}), 1.56-1.19$ (30H, env.), 0.91-0.85 (19H, m); $\delta_{\mathrm{C}}\left(75 \mathrm{MHz}, \mathrm{CDCl}_{3}\right) 121.4(\mathrm{t}$, $\left.{ }^{1} J_{\mathrm{C}-\mathrm{F}} 266.1\right), 109.2,86.2,83.9\left(\mathrm{t},{ }^{2} J_{\mathrm{C}-\mathrm{F}} 19.6\right), 81.6\left(\mathrm{t},{ }^{2} J_{\mathrm{C}-\mathrm{F}} 26.2\right)$, $63.4\left(\mathrm{t},{ }^{3} J_{\mathrm{C}-\mathrm{F}} 16.2\right), 33.2\left(\mathrm{t},{ }^{3} J_{\mathrm{C}-\mathrm{Sn}} 8.5\right), 27.4\left(\mathrm{t},{ }^{3} J_{\mathrm{C}-\mathrm{Sn}} 10.2\right), 25.8$ $\left(\mathrm{t},{ }^{2} J_{\mathrm{C}-\mathrm{Sn}} 27.2\right), 25.2,23.0,17.4\left(\mathrm{t},{ }^{1} J_{\mathrm{C}-\mathrm{Sn}} 148.9\right), 14.8,12.1,7.1(\mathrm{t}$, $\left.{ }^{1} J_{\mathrm{C}-\mathrm{Sn}} 157.4\right) ; \delta_{\mathrm{F}}\left(282 \mathrm{MHz}, \mathrm{CDCl}_{3}\right)-109.9\left(1 \mathrm{~F}, \mathrm{dd},{ }^{2} J_{\mathrm{F}-\mathrm{F}} 225.6\right.$, $\left.{ }^{3} J_{\mathrm{F}-\mathrm{H}} 9.0\right),-115.3\left(1 \mathrm{~F}, \mathrm{~d},{ }^{2} J_{\mathrm{F}-\mathrm{F}} 225.6\right)$; [HRMS (ES [M + Na $\left.]^{+}\right)$ Found 546.1946. Calc for $\mathrm{C}_{23} \mathrm{H}_{41} \mathrm{O}_{3} \mathrm{~F}_{2} \mathrm{NaSn}$ : 546.1943]; $\mathrm{m} / \mathrm{z}$ (ES) $547\left(60 \%,\left[\mathrm{M}\left({ }^{1{ }^{19}} \mathrm{Sn}\right)+\mathrm{Na}\right]^{+}\right), 549\left(100,\left[\mathrm{M}\left({ }^{119} \mathrm{Sn}\right)+\mathrm{Na}\right]^{+}\right)$. The stannane product was contaminated with traces of trialkyltin compounds which could not be removed by chromatography.

\section{3,3-Difluoro-2 $R^{*}$-(hydroxymethyl)-1-methyl-5 $R^{*}$-tributyl-} stannyl-7-oxa-1 $S^{*}, 4 S^{*}$-bicyclo[2.2.1]heptan-2-ol dimethyl acetonide 28

Was prepared as for $\mathbf{2 7}$ from acetonide $\mathbf{2 2 b}(1.5 \mathrm{mmol}, 0.35 \mathrm{~g})$, tributyltin hydride $(3.2 \mathrm{mmol}, 0.9 \mathrm{~mL}), \mathrm{Pd}_{2} \mathrm{dba}_{3} \cdot \mathrm{CHCl}_{3}(0.03$ mmol, $0.03 \mathrm{~g})$, triphenylphosphine $(0.12 \mathrm{mmol}, 0.03 \mathrm{~g})$ and toluene $(24 \mathrm{~mL})$. After stirring at room temperature for 16 hours the solvent was removed in vacuo and the resulting oil purified by column chromatography ( $5 \%$ diethyl ether in light petroleum) to afford stannane $28(0.44 \mathrm{~g}, 56 \%)$ as a colourless oil; $R_{\mathrm{f}}\left(5 \%\right.$ diethyl ether in light petroleum) $0.16 ; v_{\max }($ film)/ $\mathrm{cm}^{-1} 2959 \mathrm{~s}(\mathrm{C}-\mathrm{H}), 2928 \mathrm{~s}(\mathrm{C}-\mathrm{H}), 2874 \mathrm{~s}(\mathrm{C}-\mathrm{H}), 1457 \mathrm{~m}(\mathrm{C}-\mathrm{C})$, $1086 \mathrm{~s}(\mathrm{C}-\mathrm{O}) ; \delta_{\mathrm{H}}\left(300 \mathrm{MHz}, \mathrm{CDCl}_{3}\right) 4.26(1 \mathrm{H}, \mathrm{d}, J 1.8$, $\left.\mathrm{CHOCF}_{2}\right), 4.22\left(1 \mathrm{H}, \mathrm{d},{ }^{2} J\right.$ 9.6, $\left.\mathrm{CH}_{\mathrm{a}} \mathrm{H}_{\mathrm{b}} \mathrm{O}\right), 3.70\left(1 \mathrm{H}, \mathrm{dt},{ }^{2} J 9.6\right.$, $\left.{ }^{4} J_{\mathrm{H}-\mathrm{F}} 2.9, \mathrm{CH}_{\mathrm{a}} H_{\mathrm{b}} \mathrm{O}\right), 1.78-0.85\left(39 \mathrm{H}\right.$, env., all $n$-Bu, $\mathrm{CHCH}_{2}$, $\left.\mathrm{C} H \mathrm{Sn}, \mathrm{C}\left(\mathrm{CH}_{3}\right) \mathrm{O}, \mathrm{CH}_{3}\right) ; \delta_{\mathrm{C}}\left(75 \mathrm{MHz}, \mathrm{CDCl}_{3}\right) 125.3\left(\mathrm{dd},{ }^{1} J_{\mathrm{C}-\mathrm{F}}\right.$ $271.7,268.2), 112.2,88.5\left(\mathrm{dd},{ }^{2} J_{\mathrm{C}-\mathrm{F}} 26.0,18.0\right), 88.1,82.8(\mathrm{t}$, $\left.{ }^{2} J_{\mathrm{C}-\mathrm{F}} 26.6\right), 65.8\left(\mathrm{~d},{ }^{2} J_{\mathrm{C}-\mathrm{F}} 17.0\right), 35.6,29.0\left(\mathrm{t},{ }^{3} J_{\mathrm{C}-\mathrm{Sn}} 10.0\right), 27.4(\mathrm{t}$, $\left.{ }^{2} J_{\mathrm{C}-\mathrm{Sn}} 27.3\right), 26.1,25.7,18.7,16.0,113.6,8.8\left(\mathrm{t},{ }^{1} J_{\mathrm{C}-\mathrm{Sn}} 159.9\right)$; $\delta_{\mathrm{F}}\left(282 \mathrm{MHz}, \mathrm{CDCl}_{3}\right)-110.2\left(1 \mathrm{~F}, \mathrm{dd},{ }^{2} J_{\mathrm{F}-\mathrm{F}} 221.9,{ }^{3} J_{\mathrm{F}-\mathrm{H}} 7.6\right)$, $-118.8\left(1 \mathrm{~F}, \mathrm{~d},{ }^{2} J_{\mathrm{F}-\mathrm{F}} 221.9\right)$ [HRMS (EI, [M]+) Found 520.21237 Calc. for $\mathrm{C}_{23} \mathrm{H}_{42} \mathrm{O}_{3} \mathrm{~F}_{2}{ }^{116} \mathrm{Sn} 520.21230$ ]; $\mathrm{m} / \mathrm{z}$ (EI) 520 (8\%), 467 (66), 389 (20), 291 (68), 253 (62), 214 (78), 177 (74), $137(100)$. The stannane product was contaminated with traces of trialkyltin compounds which could not be removed by chromatography. The additional ${ }^{1} J_{\mathrm{C}-\mathrm{Sn}}$ coupling could not be detected in the ${ }^{13} \mathrm{C}$ NMR spectrum of $\mathbf{2 8}$.

\section{Ring-opening and deprotection reactions: 2,2-difluoro-3- (hydroxymethyl)cyclohex-4-ene-( $\left.1 R^{*}, 3 R^{*}\right)$-diol dimethyl acetonide 29}

Methyllithium (1.2 mmol, $1.1 \mathrm{~mL}$ of a $1.1 \mathrm{M}$ solution in diethyl ether), was added slowly to a solution of stannane 25 (1.2 mmol $0.59 \mathrm{~g})$ in THF $(11 \mathrm{~mL})$ at $0{ }^{\circ} \mathrm{C}$. The colourless solution was stirred at $0{ }^{\circ} \mathrm{C}$ for 20 minutes before being quenched with ammonium chloride ( $10 \mathrm{~mL}$ of a saturated aqueous solution) and extracted with ethyl acetate $(3 \times 15 \mathrm{~mL})$. The combined organic extracts were dried $\left(\mathrm{MgSO}_{4}\right)$ and concentrated to leave a white suspension which was triturated with hexane $(3 \times 2 \mathrm{~mL})$ to afford alcohol 29 as needles $(0.19 \mathrm{~g}, 71 \%)$; mp $73-74{ }^{\circ} \mathrm{C} ; R_{\mathrm{f}}$ (60\% diethyl ether in light petroleum) 0.3 (Found: $\mathrm{C}, 54.5 ; \mathrm{H}$, 6.3; $\mathrm{C}_{10} \mathrm{H}_{14} \mathrm{~F}_{2} \mathrm{O}_{3}$ requires: C 54.5; $\left.\mathrm{H}, 6.4 \%\right) ; v_{\max }(\mathrm{film}) / \mathrm{cm}^{-1}$ $3444 \mathrm{~m}(\mathrm{OH}), 2925 \mathrm{w}(\mathrm{C}-\mathrm{H}), 1110 \mathrm{~s}(\mathrm{C}-\mathrm{O}), 668 \mathrm{~m}(\mathrm{HC}=\mathrm{CH})$; $\delta_{\mathrm{H}}\left(300 \mathrm{MHz}, \mathrm{CDCl}_{3}\right) 5.77\left(1 \mathrm{H}, \mathrm{dt}, J 10.3,3.8,=\mathrm{CHCH}_{2}\right), 5.58-$ $5.51\left(1 \mathrm{H}, \mathrm{m}, \mathrm{HC}=\mathrm{CHCH}_{2}\right), 4.27\left(1 \mathrm{H}, \mathrm{dd},{ }^{2} J 9.3,{ }^{4} J_{\mathrm{H}-\mathrm{F}} 1.8\right.$, $\left.\mathrm{CH}_{\mathrm{a}} \mathrm{H}_{\mathrm{b}} \mathrm{O}\right), 4.08-3.95(1 \mathrm{H}, \mathrm{m}, \mathrm{CHOH}), 3.79\left(1 \mathrm{H}, \mathrm{dd},{ }^{2} J\right.$ 9.3, $\left.{ }^{4} J_{\mathrm{H}-\mathrm{F}} 1.5, \mathrm{CH}_{\mathrm{a}} H_{\mathrm{b}} \mathrm{O}\right), 3.10(1 \mathrm{H}, \mathrm{d}, J 6.3, \mathrm{OH}), 2.59-2.33(2 \mathrm{H}, \mathrm{m}$, $\left.=\mathrm{CHCH}_{2}\right), 1.46\left(3 \mathrm{H}, \mathrm{s}, \mathrm{CH}_{3}\right), 1.42\left(3 \mathrm{H}, \mathrm{s}, \mathrm{CH}_{3}\right) ; \delta_{\mathrm{C}}(75 \mathrm{MHz}$, $\left.\mathrm{CDCl}_{3}\right) 127.4\left(\mathrm{t},{ }^{3} J_{\mathrm{C}-\mathrm{F}} 2.0\right), 126.8,118.7\left(\mathrm{dd},{ }^{1} J_{\mathrm{C}-\mathrm{F}} 254.3,248.1\right)$, $79.5\left(\mathrm{dd},{ }^{2} J_{\mathrm{C}-\mathrm{F}} 26.0,20.4\right), 69.8\left(\mathrm{dd},{ }^{3} J_{\mathrm{C}-\mathrm{F}} 4.5,1.7\right), 67.7\left(\mathrm{dd},{ }^{2} J_{\mathrm{C}-\mathrm{F}}\right.$ $26.9,23.5), 65.7,32.0\left(\mathrm{dd},{ }^{3} J_{\mathrm{C}-\mathrm{F}} 4.5,2.3\right), 26.4,25.5 ; \delta_{\mathrm{F}}(282 \mathrm{MHz}$, $\left.\mathrm{CDCl}_{3}\right)-123.3\left(1 \mathrm{~F}, \mathrm{~d},{ }^{2} J_{\mathrm{F}-\mathrm{F}} 248.0\right),-128.1\left(1 \mathrm{~F}, \mathrm{~d},{ }^{2} J_{\mathrm{F}-\mathrm{F}} 248.0\right)$; $\mathrm{m} / \mathrm{z}(\mathrm{CI}) 221\left(100 \%,[\mathrm{M}+\mathrm{H}]^{+}\right), 205(6)$.

\section{3,3-Difluoro-2-(hydroxymethyl)cyclohex-4-ene-( $\left.1 S^{*}, 2 S^{*}\right)$-dio dimethyl acetonide 30}

Prepared as for 29 from stannane 26 (0.61 mmol, 0.31g), MeLi ( $0.61 \mathrm{mmol}, 0.56 \mathrm{~mL}$ of a $1.1 \mathrm{M}$ solution in diethyl ether), in THF (6 mL). Usual work-up afforded a white semi-solid which was triturated with hexane $(3 \times 2 \mathrm{~mL})$ to afford alcohol 30 as cubes $(0.05 \mathrm{~g}, 40 \%) ; \mathrm{mp} 81-83{ }^{\circ} \mathrm{C} ; R_{\mathrm{f}}(60 \%$ diethyl ether in light petroleum) 0.38 (Found: $\mathrm{C}, 54.7 ; \mathrm{H}, 6.5 ; \mathrm{C}_{10} \mathrm{H}_{14} \mathrm{~F}_{2} \mathrm{O}_{3}$ requires: $\mathrm{C}$ 54.5 ; H, 6.4\%); $v_{\max }($ film $) / \mathrm{cm}^{-1} 3476 \mathrm{~m}$ br $(\mathrm{OH}), 2996 \mathrm{~m}(\mathrm{C}-\mathrm{H})$, $2943 \mathrm{~m}(\mathrm{C}-\mathrm{H}), 1666 \mathrm{w}(\mathrm{C}=\mathrm{C}), 1165 \mathrm{~m}(\mathrm{C}-\mathrm{O}), 721 \mathrm{w}(\mathrm{HC}=\mathrm{CH})$; $\delta_{\mathrm{H}}\left(300 \mathrm{MHz}, \mathrm{CDCl}_{3}\right) 6.16-6.08\left(1 \mathrm{H}, \mathrm{m},=\mathrm{CHCH}_{2}\right), 5.80-5.72$ $\left(1 \mathrm{H}, \mathrm{m},=\mathrm{CHCF}_{2}\right), 4.37\left(1 \mathrm{H}, \mathrm{d},{ }^{2} J 9.2, \mathrm{CH}_{\mathrm{a}} \mathrm{H}_{\mathrm{b}} \mathrm{O}\right), 4.23(1 \mathrm{H}, \mathrm{d}$, $J$ 9.2, $\left.\mathrm{CH}_{\mathrm{a}} H_{\mathrm{b}} \mathrm{O}\right), 3.98-3.89(1 \mathrm{H}, \mathrm{m}, \mathrm{CHOH}), 2.58-2.46(\mathrm{~m}, 1 \mathrm{H}$, $\left.=\mathrm{CHCH}_{\mathrm{a}} \mathrm{H}_{\mathrm{b}}\right), 2.41-2.25\left(\mathrm{~m}, 1 \mathrm{H},=\mathrm{CHCH}_{\mathrm{a}} H_{\mathrm{b}}\right), 1.90(\mathrm{~d}, 1 \mathrm{H}$, $J 8.5, \mathrm{OH}), 1.50\left(3 \mathrm{H}, \mathrm{s}, \mathrm{CH}_{3}\right), 1.46\left(3 \mathrm{H}, \mathrm{s}, \mathrm{CH}_{3}\right) ; \delta_{\mathrm{C}}(75 \mathrm{MHz}$, 
$\left.\mathrm{CDCl}_{3}\right) 134.6\left(\mathrm{dd},{ }^{3} J_{\mathrm{C}-\mathrm{F}} 13.0,10.2\right), 122.3\left(\mathrm{dd},{ }^{2} J_{\mathrm{C}-\mathrm{F}} 32.8,24.3\right)$, $118.0\left(\mathrm{dd},{ }^{1} J_{\mathrm{C}-\mathrm{F}} 247.0,235.1\right), 112.0,83.1\left(\mathrm{dd},{ }^{2} J_{\mathrm{C}-\mathrm{F}} 29.4,19.2\right)$, $68.4\left(\mathrm{~d},{ }^{3} J_{\mathrm{C}-\mathrm{F}} 4,5\right), 65.5\left(\mathrm{dd},{ }^{3} J_{\mathrm{C}-\mathrm{F}} 4.5,1.4\right), 31.9\left(\mathrm{t},{ }^{4} J_{\mathrm{C}-\mathrm{F}} 2.0\right)$, $27.0,25.1 ; \delta_{\mathrm{F}}\left(282 \mathrm{MHz}, \mathrm{CDCl}_{3}\right)-90.2\left(1 \mathrm{~F}, \mathrm{~d},{ }^{2} J_{\mathrm{F}-\mathrm{F}} 282.9\right)$, $-111.5\left(1 \mathrm{~F}, \mathrm{~d},{ }^{2} J_{\mathrm{F}-\mathrm{F}} 282.9\right) ; \mathrm{m} / z(\mathrm{CI}) 238\left(7 \%,\left[\mathrm{M}+\mathrm{NH}_{4}\right]^{+}\right), 221$ $\left([\mathrm{M}+\mathrm{H}]^{+}, 100\right), 205(7)$.

\section{3,3-Difluoro-2-(hydroxymethyl)-1-methylcyclohex-4-ene- $\left(1 S^{*}, 2 S^{*}\right)$-diol dimethyl acetonide 31}

Prepared as for 29 from stannane 27 ( $0.84 \mathrm{mmol}, 0.44 \mathrm{~g})$, methyllithium $(0.84 \mathrm{mmol}, 0.76 \mathrm{~mL}$ of a $1.1 \mathrm{M}$ solution in diethyl ether) and THF $(8 \mathrm{~mL})$ at $0{ }^{\circ} \mathrm{C}$ to afford a colourless oil. Purification by column chromatography $(10 \%$ diethyl ether in light petroleum) yielded 31 as needles $\left(0.06 \mathrm{~g}, 30 \%, 100 \%\right.$ by GC); $R_{\mathrm{f}}$ $\left(10 \%\right.$ diethyl ether in light petroleum) $0.05 ; \mathrm{mp} 38-40{ }^{\circ} \mathrm{C} ; v_{\max }$ (film) $/ \mathrm{cm}^{-1} 3458 \mathrm{~m}$ br $(\mathrm{OH}), 2991 \mathrm{~m}(\mathrm{C}-\mathrm{H}), 2937 \mathrm{~m}(\mathrm{C}-\mathrm{H})$, $2360 \mathrm{~m}, 1060 \mathrm{~s}(\mathrm{C}-\mathrm{O}), 1031 \mathrm{~s}(\mathrm{C}-\mathrm{O}) ; \delta_{\mathrm{H}}\left(300 \mathrm{MHz}, \mathrm{CDCl}_{3}\right) 6.11-$ $6.05\left(1 \mathrm{H}, \mathrm{m},=\mathrm{CHCH}_{2}\right), 5.82-5.75\left(1 \mathrm{H}, \mathrm{m},=\mathrm{CHCF}_{2}\right), 4.31(1 \mathrm{H}$, d, $\left.{ }^{2} J 9.4, \mathrm{CH}_{\mathrm{a}} \mathrm{H}_{\mathrm{b}} \mathrm{O}\right), 4.17\left(1 \mathrm{H}, \mathrm{d},{ }^{2} J 9.4, \mathrm{CH}_{\mathrm{a}} H_{\mathrm{b}} \mathrm{O}\right), 2.51-2.43$ $\left(1 \mathrm{H}, \mathrm{m},=\mathrm{CHCH}_{\mathrm{a}} \mathrm{H}_{\mathrm{b}}\right), 2.23-2.17\left(1 \mathrm{H}, \mathrm{m},=\mathrm{CHCH}_{\mathrm{a}} H_{\mathrm{b}}\right), 1.47$ $\left(6 \mathrm{H}, \mathrm{s}, \mathrm{C}\left(\mathrm{CH}_{3}\right)_{2}\right), 1.35\left(3 \mathrm{H}, \mathrm{s}, \mathrm{C}\left(\mathrm{CH}_{3}\right) \mathrm{OH}\right) ; \delta_{\mathrm{C}}\left(75 \mathrm{MHz}, \mathrm{CDCl}_{3}\right)$ $135.1\left(\mathrm{t},{ }^{3} J_{\mathrm{C}-\mathrm{F}} 11.4\right), 122.4\left(\mathrm{dd},{ }^{2} J_{\mathrm{C}-\mathrm{F}} 31.9,25.3\right), 118.3\left(\mathrm{dd},{ }^{1} J_{\mathrm{C}-\mathrm{F}}\right.$ $245.0,237.2), 112.5,86.2\left(\mathrm{dd},{ }^{2} J_{\mathrm{C}-\mathrm{F}} 27.7,18.1\right), 71.8\left(\mathrm{~d},{ }^{3} J_{\mathrm{C}-\mathrm{F}}\right.$ $4.2), 64.6\left(\mathrm{~d},{ }^{3} J_{\mathrm{C}-\mathrm{F}} 6.0\right), 39.6,27.1,26.0,23.0 ; \delta_{\mathrm{F}}(282 \mathrm{MHz}$ $\left.\mathrm{CDCl}_{3}\right)-98.8\left(1 \mathrm{~F}, \mathrm{~d},{ }^{2} J_{\mathrm{F}-\mathrm{F}} 284.8\right),-102.6\left(1 \mathrm{~F}, \mathrm{~d},{ }^{2} J_{\mathrm{F}-\mathrm{F}} 284.8\right)$ [HRMS (ES, $[\mathrm{M}+\mathrm{Na}]^{+}$) Found 257.0959. Calc. for $\mathrm{C}_{11} \mathrm{H}_{16}$ $\left.\mathrm{O}_{3} \mathrm{~F}_{2} \mathrm{Na} 257.0965\right] ; \mathrm{m} / z$ (ES) $257\left(100 \%[\mathrm{M}+\mathrm{Na}]^{+}\right)$. Satisfactory microanalysis could not be obtained for this compound after a number of attempts. Potential contamination with organotin compounds would prevent accurate combustion analysis.

\section{3,3-Difluoro-2-(hydroxymethyl)-1-methylcyclohex-4-ene-} $\left(1 S^{*}, 2 R^{*}\right)$-diol dimethyl acetonide 32

Prepared as for 29 from stannane 28 (0.9 mmol, $0.48 \mathrm{~g})$, methyllithium $(1.0 \mathrm{mmol}, 0.9 \mathrm{~mL}$ of a $1.1 \mathrm{M}$ solution in diethyl ether) and THF $(8 \mathrm{~mL})$ at $0{ }^{\circ} \mathrm{C}$. Usual work-up afforded a colourless oil. Purification by column chromatography $(10 \%$ diethyl ether in light petroleum) yielded 32 as needles $(0.136 \mathrm{~g}, 65 \%, 100 \%$ by $\mathrm{GC}) ; R_{\mathrm{f}}\left(40 \%\right.$ diethyl ether in light petroleum) $0.3 ; \mathrm{mp} 33-35^{\circ} \mathrm{C}$; $v_{\max }($ film $) / \mathrm{cm}^{-1} 3474 \mathrm{~m}$ br $(\mathrm{OH}), 2989 \mathrm{~m}(\mathrm{C}-\mathrm{H}), 2940(\mathrm{C}=\mathrm{H})$, $1666 \mathrm{w}(\mathrm{C}=\mathrm{C}), 1169 \mathrm{~m}(\mathrm{C}-\mathrm{O}), 1107 \mathrm{~m}(\mathrm{C}-\mathrm{O}) ; \delta_{\mathrm{H}}\left(300 \mathrm{MHz}, \mathrm{CDCl}_{3}\right)$ 6.12-6.05 $\left(1 \mathrm{H}, \mathrm{m},=\mathrm{CHCH}_{2}\right), 5.75-5.67\left(1 \mathrm{H}, \mathrm{m},=\mathrm{CHCF}_{2}\right), 4.37$ $\left(1 \mathrm{H}, \mathrm{dd},{ }^{2} J 9.2,{ }^{4} J_{\mathrm{H}-\mathrm{F}} 1.0\right), 4.20\left(1 \mathrm{H}, \mathrm{dd},{ }^{2} J 9.2,{ }^{4} J_{\mathrm{H}-\mathrm{F}} 1.0\right), 2.48$ $2.39\left(1 \mathrm{H}, \mathrm{m},=\mathrm{CHCH}_{\mathrm{a}} \mathrm{H}_{\mathrm{b}}\right), 2.28-2.24\left(1 \mathrm{H}, \mathrm{m},=\mathrm{CHCH}_{\mathrm{a}} H_{\mathrm{b}}\right), 2.22$ $2.18(1 \mathrm{H}, \mathrm{m}, \mathrm{OH}), 1.46\left(6 \mathrm{H}, \mathrm{s}, \mathrm{C}\left(\mathrm{CH}_{3}\right)_{2}\right), 1.20\left(3 \mathrm{H}, \mathrm{s}, \mathrm{C}\left(\mathrm{CH}_{3}\right)-\right.$ $\mathrm{OH}) ; \delta_{\mathrm{C}}\left(75 \mathrm{MHz}, \mathrm{CDCl}_{3}\right) 134.7\left(\mathrm{dd},{ }^{3} J_{\mathrm{C}-\mathrm{F}} 12.4,10.7\right), 122.0(\mathrm{dd}$, $\left.{ }^{2} J_{\mathrm{C}-\mathrm{F}} 31.7,25.4\right), 117.9\left(\mathrm{dd},{ }^{1} J_{\mathrm{C}-\mathrm{F}} 245.3,236.8\right), 85.8\left(\mathrm{dd},{ }^{2} J_{\mathrm{C}-\mathrm{F}}\right.$ $27.7,18.1), 71.5\left(\mathrm{~d},{ }^{3} J_{\mathrm{C}-\mathrm{F}} 4.5\right), 64.2\left(\mathrm{~d},{ }^{3} J_{\mathrm{C}-\mathrm{F}} 6.8\right), 39.2\left(\mathrm{t},{ }^{4} J_{\mathrm{C}-\mathrm{F}}\right.$ 2.0), 26.7, 25.6, 22.6; $\delta_{\mathrm{F}}\left(282 \mathrm{MHz}, \mathrm{CDCl}_{3}\right)-92.9\left(1 \mathrm{~F}, \mathrm{~d},{ }^{2} J_{\mathrm{F}-\mathrm{F}}\right.$ 286.7), -105.1 (1F, d, ${ }^{2} J_{\mathrm{F}-\mathrm{F}}$ 286.7) [HRMS (EI, [M] $\left.{ }^{+}\right)$Found 234.1067. Calc. for $\mathrm{C}_{11} \mathrm{H}_{16} \mathrm{O}_{3} \mathrm{~F}_{2}$ 234.1068]; $\mathrm{m} / z$ (EI) $234(15 \%), 219$ (100), 176 (18), 127 (41), 113 (40), 97 (45). Satisfactory microanalysis could not be obtained for this compound after a number of attempts. Potential contamination with organotin compounds would prevent accurate combustion analysis.

\section{Deprotection: 2,2-difluoro-3-hydroxymethylcyclohex-4-ene- $\left(1 R^{*}, 3 R^{*}\right)$-diol 33}

Amberlyst-15 $(0.08 \mathrm{~g})$ was added to a solution of acetonide 29 $(0.2 \mathrm{mmol}, 0.04 \mathrm{~g})$ in methanol $(2 \mathrm{~mL})$ at $40{ }^{\circ} \mathrm{C}$. After heating for 24 hours, the Amberlyst was removed by filtration and washed with methanol $(2 \times 2 \mathrm{~mL})$, combining filtrate and washings. The combined methanol phases were concentrated in vacuo to afford triol 33 as needles $(0.03 \mathrm{~g}, 93 \%) ; R_{\mathrm{f}}(80 \%$ ethyl acetate in light petroleum) 0.08 ; $\mathrm{mp} 138-140{ }^{\circ} \mathrm{C}$ (Found: $\mathrm{C}$, 46.50; $\mathrm{H}, 5.55 ; \mathrm{C}_{7} \mathrm{H}_{10} \mathrm{~F}_{2} \mathrm{O}_{3}$ requires: $\mathrm{C} 46.67 ; \mathrm{H}, 5.60 \%$; $v_{\max }($ solid $) / \mathrm{cm}^{-1} 3287 \mathrm{br}(\mathrm{OH}), 1282 \mathrm{w}(\mathrm{C}-\mathrm{O}), 1216 \mathrm{~m}(\mathrm{C}-\mathrm{O})$, $1667 \mathrm{w}(\mathrm{C}=\mathrm{C}) ; \delta_{\mathrm{H}}\left(400 \mathrm{MHz}, \mathrm{CD}_{3} \mathrm{OD}\right) 5.83(1 \mathrm{H}, \mathrm{ddd}, J 10.3$,
5.0, 2.5, $\left.=\mathrm{CHCH}_{2}\right), 5.48-5.43(1 \mathrm{H}, \mathrm{m},=\mathrm{CHCHOH}), 4.36(1 \mathrm{H}$, dddd, $\left.{ }^{3} J_{\mathrm{H}-\mathrm{F}} 16.4, J 10.0,9.1,6.7, \mathrm{CHOH}\right), 3.67\left(1 \mathrm{H}, \mathrm{d},{ }^{2} J 11.4\right.$, $\left.\mathrm{C}_{\mathrm{a}} \mathrm{H}_{\mathrm{b}} \mathrm{O}\right), 3.51\left(1 \mathrm{H}, \mathrm{dt},{ }^{2} J 11.4,{ }^{4} J_{\mathrm{H}-\mathrm{F}} 2.7, \mathrm{CH}_{\mathrm{a}} H_{\mathrm{b}} \mathrm{O}\right), 2.57-$ $2.49\left(1 \mathrm{H}, \mathrm{m},=\mathrm{CHCH}_{\mathrm{a}} \mathrm{H}_{\mathrm{b}}\right), 2.27(1 \mathrm{H}, \mathrm{ddt}, J 17.6,9.1,2.7$, $\left.=\mathrm{CHCH}_{\mathrm{a}} H_{\mathrm{b}}\right) ; \delta_{\mathrm{C}}\left(100 \mathrm{MHz}, \mathrm{CD}_{3} \mathrm{OD}\right) 129.2,127.1,121.0(\mathrm{t}$, $\left.{ }^{1} J_{\mathrm{C}-\mathrm{F}} 249.0\right), 75.0\left(\mathrm{t},{ }^{2} J_{\mathrm{C}-\mathrm{F}} 20.9\right), 67.2\left(\mathrm{t},{ }^{2} J_{\mathrm{C}-\mathrm{F}} 21.8\right), 64.8\left(\mathrm{t},{ }^{3} J_{\mathrm{C}-\mathrm{F}}\right.$ $3.7), 32.1\left(\mathrm{~d},{ }^{3} J_{\mathrm{C}-\mathrm{F}} 3.6\right) ; \delta_{\mathrm{F}}\left(376 \mathrm{MHz}, \mathrm{CD}_{3} \mathrm{OD}\right)-130.58(1 \mathrm{~F}, \mathrm{~d}$, $\left.{ }^{3} J_{\mathrm{H}-\mathrm{F}} 16.4\right),-130.60(1 \mathrm{~F}, \mathrm{~s})$ [HRMS (FAB, [M $\left.\left.-\mathrm{H}\right]^{-}\right)$Found 179.05187. Calc. for $\mathrm{C}_{7} \mathrm{H}_{9} \mathrm{O}_{3} \mathrm{~F}_{2}$ 179.05198]; $\mathrm{m} / \mathrm{z}(\mathrm{FAB}) 179(10 \%$ $\left.[\mathrm{M}-\mathrm{H}]^{-}\right)$. See text for comments on the appearance of this ${ }^{19} \mathrm{~F}$ NMR spectrum. The proton labelled $=\mathrm{CHCH}_{\mathrm{a}} H_{\mathrm{b}}$ is believed to be pseudo-axial.

\section{6,6-Difluoro-1-hydroxymethylcyclohex-4-ene-( $\left.1 S^{*}, 2 S^{*}\right)$-diol 34}

Prepared as for $\mathbf{3 3}$ from acetonide $\mathbf{3 1}(0.07 \mathrm{mmol}, 0.02 \mathrm{~g})$, Amberlyst-15 $(0.04 \mathrm{~g})$ and methanol $(1 \mathrm{~mL})$. After heating for 24 hours at $40{ }^{\circ} \mathrm{C}$, the usual work-up afforded triol $\mathbf{3 4}$ as needles $(0.012 \mathrm{~g}$, $95 \%) ; R_{\mathrm{f}}(80 \%$ ethyl acetate in light petroleum) $0.13 ; \mathrm{mp} 100$ $103{ }^{\circ} \mathrm{C}$ (Found: $\mathrm{C}, 46.42 ; \mathrm{H}, 5.43 ; \mathrm{C}_{7} \mathrm{H}_{10} \mathrm{~F}_{2} \mathrm{O}_{3}$ requires: $\mathrm{C} 46.67$; $\mathrm{H}, 5.60 \%) ; v_{\max }$ (solid) $/ \mathrm{cm}^{-1} 3385 \mathrm{br}(\mathrm{OH}), 1275 \mathrm{~m}(\mathrm{C}-\mathrm{O}), 1200 \mathrm{~m}$ $(\mathrm{C}-\mathrm{O}), 1659 \mathrm{~m}(\mathrm{C}=\mathrm{C}), 2933 \mathrm{w}(\mathrm{C}-\mathrm{H}) ; \delta_{\mathrm{H}}\left(400 \mathrm{MHz}, \mathrm{CD}_{3} \mathrm{OD}\right)$ 6.15-6.10 $\left(1 \mathrm{H}, \mathrm{m},=\mathrm{CHCH}_{2}\right), 5.70-5.64\left(1 \mathrm{H}, \mathrm{m},=\mathrm{CHCF}_{2}\right), 4.06-$ $4.01(1 \mathrm{H}, \mathrm{m}, \mathrm{CHOH}), 3.96\left(1 \mathrm{H}, \mathrm{dd},{ }^{2} J 12.0,{ }^{4} J_{\mathrm{H}-\mathrm{F}} 1.8, \mathrm{CH}_{\mathrm{a}} \mathrm{H}_{\mathrm{b}} \mathrm{O}\right)$, $3.88\left(1 \mathrm{H}, \mathrm{dd},{ }^{2} J 12.0,{ }^{4} J_{\mathrm{H}-\mathrm{F}} 1.0, \mathrm{CH}_{\mathrm{a}} H_{\mathrm{b}} \mathrm{O}\right), 2.44-2.38(2 \mathrm{H}, \mathrm{m}$, $\left.=\mathrm{CHCH}_{2}\right) ; \delta_{\mathrm{C}}\left(75 \mathrm{MHz}, \mathrm{CD}_{3} \mathrm{OD}\right) 134.4\left(\mathrm{t},{ }^{3} J_{\mathrm{C}-\mathrm{F}} 11.5\right), 122.2(\mathrm{dd}$, $\left.{ }^{2} J_{\mathrm{C}-\mathrm{F}} 31.9,25.3\right), 120.1\left(\mathrm{dd}^{1} J_{\mathrm{C}-\mathrm{F}} 243.3,237.1\right), 74.5\left(\mathrm{dd},{ }^{2} J_{\mathrm{C}-\mathrm{F}} 24.3\right.$, $18.8), 68.5\left(\mathrm{~d},{ }^{3} J_{\mathrm{C}-\mathrm{F}} 3.6\right), 61.9\left(\mathrm{~d},{ }^{3} J_{\mathrm{C}-\mathrm{F}} 4.9\right), 30.9 ; \delta_{\mathrm{F}}(282 \mathrm{MHz}$, $\left.\mathrm{CD}_{3} \mathrm{OD}\right)-97.0\left(1 \mathrm{~F}, \mathrm{~d},{ }^{2} J_{\mathrm{F}-\mathrm{F}} 281.8\right),-113.1\left(1 \mathrm{~F}, \mathrm{dd},{ }^{2} J_{\mathrm{F}-\mathrm{F}} 281.8\right.$, ${ }^{3} J_{\mathrm{H}-\mathrm{F}}$ 7.7) $\left[\mathrm{HRMS}\left(\mathrm{FAB},[\mathrm{M}-\mathrm{H}]^{-}\right)\right.$Found 179.05186. Calc. for $\mathrm{C}_{7} \mathrm{H}_{9} \mathrm{O}_{3} \mathrm{~F}_{2}$ 179.05198]; $m / z(\mathrm{FAB}) 179\left(11 \%[\mathrm{M}-\mathrm{H}]^{-}\right)$.

\section{6,6-Difluoro-1-(hydroxymethyl)-2-methyl cyclohex-4-ene- $\left(1 S^{*}, 2 S^{*}\right)$-diol 35}

Was prepared as for $\mathbf{3 3}$ from acetonide $\mathbf{3 1}(0.21 \mathrm{mmol}, 0.05 \mathrm{~g})$, Amberlyst-15 $(0.04 \mathrm{~g})$ in methanol $(2 \mathrm{~mL})$. Usual work-up afforded triol 35 as white needles $(0.035 \mathrm{~g}, 86 \%) ; R_{\mathrm{f}}(80 \%$ ethyl acetate in light petroleum) $0.1 ; \mathrm{mp} 58-61^{\circ} \mathrm{C}$ (Found: C, 49.32; $\mathrm{H}, 6.04 ; \mathrm{C}_{8} \mathrm{H}_{12} \mathrm{~F}_{2} \mathrm{O}_{3}$ requires: $\mathrm{C} 49.48 ; \mathrm{H}, 6.23 \%$ ); $v_{\max }$ (solution)/ $\left.\mathrm{cm}^{-1} 3320 \mathrm{br}(\mathrm{OH}), 2920 \mathrm{w}(\mathrm{C}-\mathrm{H}), 1280 \mathrm{w}(\mathrm{C}-\mathrm{O}), 1210 \mathrm{~m} \mathrm{C}-\mathrm{O}\right)$, $1663 \mathrm{w}(\mathrm{C}=\mathrm{C}) ; \delta_{\mathrm{H}}\left(300 \mathrm{MHz}, \mathrm{CD}_{3} \mathrm{OD}\right) 5.98(1 \mathrm{H}, \mathrm{dt}, J 10.4,3.8$, $\left.=\mathrm{CHCH}_{2}\right), 5.63-5.54\left(1 \mathrm{H}, \mathrm{m},=\mathrm{C} H \mathrm{CF}_{2}\right), 3.94\left(1 \mathrm{H}, \mathrm{d},{ }^{2} J 11.7\right.$, $\left.\mathrm{CH}_{\mathrm{a}} \mathrm{H}_{\mathrm{b}} \mathrm{O}\right), 3.78\left(1 \mathrm{H}, \mathrm{d},{ }^{2} J 11.7, \mathrm{CH}_{\mathrm{a}} H_{\mathrm{b}} \mathrm{O}\right), 2.41-2.29(1 \mathrm{H}, \mathrm{m}$, $\left.=\mathrm{CHCH}_{\mathrm{a}} \mathrm{H}_{\mathrm{b}}\right), 2.10-1.99\left(1 \mathrm{H}, \mathrm{m},=\mathrm{CHCH}_{\mathrm{a}} H_{\mathrm{b}}\right), 1.24(3 \mathrm{H}, \mathrm{s}$, $\left.\mathrm{CH}_{3}\right) ; \delta_{\mathrm{C}}\left(75 \mathrm{MHz}, \mathrm{CD}_{3} \mathrm{OD}\right) 135.2$, (t, $\left.{ }^{3} J_{\mathrm{C}-\mathrm{F}} 11.3\right), 123.1\left(\mathrm{t},{ }^{2} J_{\mathrm{C}-\mathrm{F}}\right.$ $28.7), 120.7\left(\mathrm{t},{ }^{1} J_{\mathrm{C}-\mathrm{F}} 239.7\right), 75.2\left(\mathrm{t},{ }^{2} J_{\mathrm{C}-\mathrm{F}} 19.6\right), 75.3,62.5(\mathrm{~d}$, $\left.{ }^{3} J_{\mathrm{C}-\mathrm{F}} 5.3\right), 39.0,24.3 ; \delta_{\mathrm{F}}\left(282 \mathrm{MHz}, \mathrm{CD}_{3} \mathrm{OD}\right)-101.3\left(\mathrm{~d},{ }^{2} J_{\mathrm{F}-\mathrm{F}}\right.$ 285.6), - 106.45 (d, ${ }^{2} J_{\mathrm{F}-\mathrm{F}}$ 285.6) [HRMS (EI, [M]+) Found 194.07549 Calc. for $\mathrm{C}_{8} \mathrm{H}_{12} \mathrm{O}_{3} \mathrm{~F}_{2}$ 194.07545]; $\mathrm{m} / \mathrm{z}$ (CI) $212(100 \%$, $\left.\left[\mathrm{M}+\mathrm{NH}_{4}\right]^{+}\right), 174(26), 133(29), 113(58), 97$ (26).

\section{6,6-Difluoro-1-hydroxymethyl-2-methyl cyclohex-4-ene- $\left(1 S^{*}, 2 R^{*}\right)$-diol 36}

Was prepared as for 33 from acetonide $32(0.14 \mathrm{mmol}, 0.03 \mathrm{~g})$, Amberlyst-15 (0.04 g) in methanol $(2 \mathrm{~mL})$. Usual work-up afforded triol 36 as an oil $(0.022 \mathrm{~g}, 92 \%) ; R_{\mathrm{f}}(80 \%$ ethyl acetate in light petroleum) $0.12 ; v_{\max }$ (solution) $/ \mathrm{cm}^{-1} 3295 \mathrm{br}(\mathrm{OH})$, 2928w (C-H), 1285w (C-O), 1205m C-O), 1670w (C=C); $\delta_{\mathrm{H}}\left(300 \mathrm{MHz}, \mathrm{CD}_{3} \mathrm{OD}\right) 6.16-6.10\left(1 \mathrm{H}, \mathrm{m},=\mathrm{CHCH}_{2}\right), 5.75-5.62$ $\left(1 \mathrm{H}, \mathrm{m},=\mathrm{CHCF}_{2}\right), 4.02-4.01\left(2 \mathrm{H}, \mathrm{m}, \mathrm{CH}_{2} \mathrm{OH}\right), 2.67-2.58(1 \mathrm{H}$, $\left.\mathrm{m},=\mathrm{CHCH}_{\mathrm{a}} \mathrm{H}_{\mathrm{b}}\right), 2.17-2.08\left(1 \mathrm{H}, \mathrm{m},=\mathrm{CHCH}_{\mathrm{a}} H_{\mathrm{b}}\right), 1.26-1.24$ $\left(3 \mathrm{H}, \mathrm{m}, \mathrm{CH}_{3}\right) * ; \delta_{\mathrm{C}}\left(75 \mathrm{MHz}, \mathrm{CD}_{3} \mathrm{OD}\right) 135.8\left(\mathrm{t},{ }^{3} J_{\mathrm{C}-\mathrm{F}} 12.1\right)$, $122.8\left(\mathrm{dd},{ }^{2} J_{\mathrm{C}-\mathrm{F}} 31.0,25.7\right), 122.6\left(\mathrm{t},{ }^{1} J_{\mathrm{C}-\mathrm{F}} 237.8\right), 76.8\left(\mathrm{t},{ }^{2} J_{\mathrm{C}-\mathrm{F}}\right.$ $21.4), 74.5,60.7\left(\mathrm{~d},{ }^{3} J_{\mathrm{C}-\mathrm{F}} 6.8\right), 39.4,23.2 ; \delta_{\mathrm{F}}\left(282 \mathrm{MHz}, \mathrm{CD}_{3} \mathrm{OD}\right)$ $-96.8\left(\mathrm{~d},{ }^{2} J_{\mathrm{F}-\mathrm{F}} 285.2\right),-108.0\left(\mathrm{~d},{ }^{2} J_{\mathrm{F}-\mathrm{F}} 285.2\right)$ [HRMS (EI, $[\mathrm{M}]+)$ Found 194.07547 Calc. for $\mathrm{C}_{8} \mathrm{H}_{12} \mathrm{O}_{3} \mathrm{~F}_{2}$ 194.07545]; $\mathrm{m} / \mathrm{z}$ (CI) $212\left(100 \%,\left[\mathrm{M}+\mathrm{NH}_{4}\right]^{+}\right), 174(23), 133(34), 114$ (79), 104 (51). *The COSY experiment detected a cross-peak between the methyl group and the more complex methylene proton methyl 
group at 2.67-2.58 ppm consistent with the presence of a ${ }^{4} \mathrm{~J}$ coupling. Unfortunately, the methyl signal could not be resolved.

\section{Acknowledgements}

We thank the Engineering and Physical Sciences Research Council of the UK for project grants GR/M94922 (studentship to VS, fellowship to AA, ACM), 98XR20 (X-ray crystallographic support) and the Department of Trade and Industry and Syngenta for an Industrial CASE Award (studentship to ACM).

\section{References}

1 T. Eguchi, S. Sasaki, Z. Huang and K. Kakinuma, J. Org. Chem., 2002, 67, 3979-3984; D. A. Sawyer and B. V. L. Potter, Bioorg. Med. Chem. Lett., 1991, 1, 705-710; D. A. Sawyer and B. V. L. Potter, J. Chem. Soc., Perkin Trans. 1, 1992, 923-932; G. D. Prestwich and J. F. Marecek, ACS Symp. Ser., 1991, 463, 122-131; D. Lampe, C. S. Liu, M. F. Mahon and B. V. L. Potter, J. Chem. Soc., Perkin Trans. 1, 1996, 1717-1727; A. P. Kozikowski, A. H. Fauq, R. A. Wilcox and S. R. Nahorski, Bioorg. Med. Chem. Lett., 1995, 5, 1295-1300; A. H. Fauq, J. H. Zaidi, R. A. Wilcox, G. Varvel, S. R. Nahorski, A. P. Kozikowski and C. Erneux, Tetrahedron Lett., 1996, 37, 1917-1920. For a review, see: B. V. L. Potter and D. Lampe, Angew. Chem., Int. Ed. Engl., 1995, 34, 1933-1972.

2 G. S. Lal, G. P. Pez, R. J. Pesaresi, F. M. Prozonic and H. S. Cheng, J. Org. Chem., 1999, 64, 7048-7054. For a recent review, see : R. P. Singh and J. M. Shreeve, Synthesis, 2002, 2561-2578. For a recent large scale comparative study of the two reagents, see: T. Mase, I. N. Houpis, A. Akao, I. Dorziotis, K. Emerson, T. Hoang, T. Iida, T. Itoh, K. Kamei, S. Kato, Y. Kato, M. Kawasaki, F. R. Lang, J. M. Lee, J. Lynch, P. Maligres, A. Molina, T. Nemoto, S. Okada, R. Reamer, J. Z. Song, D. Tschaen, T. Wada, D. Zewge, R. P. Volante, P. J. Reider and K. Tomimoto, J. Org. Chem., 2001, 66, 6775-6786.

3 R. H. Rich, B. M. Lawrence and P. A. Bartlett, J. Org. Chem., 1994, 59, 693-694; J. M. Box, L. M. Harwood, J. L. Humphreys, G. A. Morris, P. M. Redon and R. C. Whitehead, Synlett, 2002, 358-360; J. M. Box, L. M. Harwood and R. C. Whitehead, Synlett, 1997, 571573; S. Jiang, G. Singh, D. J. Boam and J. R. Coggins, Tetrahedron: Asymmetry, 1999, 10, 4087-4090; C. J. Song, S. D. Jiang and G. Singh, Tetrahedron Lett., 2001, 42, 9069-9071.

4 A. P. Kozikowski, G. Powis, A. H. Fauq, W. Tuckmantel and A. Gallegos, J. Org. Chem., 1994, 59, 963-971.

5 K. Dax and M. Albert, Top. Curr. Chem., 2001, 215, 193-275.

6 M. L. Aghmiz, Y. Diaz, G. H. Jana, M. I. Matheu, R. Echarri, S. Castillon and M. L. Jimeno, Tetrahedron, 2001, 57, 6733-6743; R. Fernandez and S. Castillon, Tetrahedron, 1999, 55, 8497-8508; M. I. Barrena, M. I. Matheu and S. Castillon, J. Org. Chem., 1998 , 63, 2184-2188; R. Fernandez, M. I. Matheu, R. Echarri and S. Castillon, Tetrahedron, 1998, 54, 3523-3532.

7 F. Barth and C. O-Yang, Tetrahedron Lett., 1991, 32, 5873-5876; A. Arnone, P. Bravo, G. Cavicchio, M. Frigerio and F. Viani, Tetrahedron, 1992, 48, 8523-8540; A. Arnone, P. Bravo, F. Viani, M. Zanda, G. Cavicchio and M. Crucianelli, J. Fluorine Chem., 1996, 76, 169-173; T. Morikawa, Y. Kodama, J. Uchida, M. Takano, Y. Washio and T. Taguchi, Tetrahedron, 1992, 48, 8915-8926.

8 D. Saleur, T. Brigaud, J. P. Bouillon and C. Portella, Synlett, 1999 , 432-434.

9 H. Ito, A. Saito and T. Taguchi, Tetrahedron: Asymmetry, 1998, 9, 1979-1987; H. Ito, A. Saito and T. Taguchi, Tetrahedron: Asymmetry, 1998, 9, 1989-1994; M. Essers and G. Haufe, J. Chem. Soc., Perkin Trans. 1, 2002, 2719-2728; T. Ernet, A. H. Maulitz, E. U. Wurthwein and G. Haufe, J. Chem. Soc., Perkin Trans. 1, 2001, 1929-1938; M. Essers, B. Wibbeling and G. Haufe, Tetrahedron Lett., 2001, 42, 5429-5433; P. J. Crowley, J. M. Percy and K. Stansfield, Tetrahedron Lett., 1996, 37, 8237-8240; T. Hanamoto, K. Korekoda, K. Nakata, K. Handa, Y. Koga and M. Kondo, J. Fluorine Chem., 2002, 118, 99-101.

10 F. Q. Jin, Y. Y. Xu and W. Y. Huang, J. Fluorine Chem., 1995, 71, 1-4 describes Diels-Alder chemistry of a difluorinated diene.

11 P. J. Crowley, J. M. Percy and K. Stansfield, Tetrahedron Lett., 1996 , 37, 8233-8236; P. J. Crowley, J. M. Percy and K. Stansfield, Chem. Commun., 1997, 2033-2034; M. Sridhar, K. L. Krishna and J. M. Rao, Tetrahedron, 2000, 56, 3539-3545.

12 C. R. Brundle, M. B. Robin, N. A. Kuebler and H. Basch, J. Am Chem. Soc., 1972, 94, 1451-1465.
13 M. Essers, C. Muck-Lichtenfeld and G. Haufe, J. Org. Chem., 2002, 67, 4715-4721 describes the application of DFT methods.

14 I. Fleming, Frontier Orbitals and Organic Chemical Reactions, J Wiley and Sons, Chichester, 1976; . For a more complex treatment of Diels-Alder reactant pairing, see : L. R. Domingo, M. J. Aurell, P. Perez and R. Contreras, Tetrahedron, 2002, 58, 4417-4423.

15 J. Ichikawa, N. Yokota, M. Kobayashi and T. Minami, Synlett, 1993 , 186-188; J. Ichikawa, M. Kobayashi, N. Yokota, Y. Noda and T. Minami, Tetrahedron, 1994, 50, 11637-11646.

16 J. Leroy, H. Molines and C. Wakselman, J. Org. Chem., 1987, 52, 290-292.

17 F. Brion, Tetrahedron Lett., 1982, 23, 5299-5302.

18 (a) P. J. Crowley, A. C. Moralee, J. M. Percy and N. S. Spencer, Synlett, 2000, 1737-1740 describes our preliminary results in this area; (b) P. J. Crowley, J. Fawcett, B. M. Kariuki, A. C. Moralee, J. M. Percy and V. Salafia, Org. Lett., 2002, 4, 4125-4128 describes, in preliminary form, an alternative ring-opening strategy.

19 H. Mayr, B. Kempf and A. R. Ofial, Acc. Chem. Res., 2003, 36, 66-77.

20 For reviews of this extremely productive area of science, see P. Chiu and M. Lautens, Top. Curr. Chem., 1997, 190, 1-85; P. Vogel, J. Cossy, J. Plumet and O. Arjona, Tetrahedron, 1999, 55, 1352113642.

21 J. A. Howarth, W. M. Owton, J. M. Percy and M. H. Rock, Tetrahedron, 1995, 51, 10289-10302.

22 V. Farina, S. Kapadia, B. Krishnan, C. Wang and L. S. Liebeskind, J. Org. Chem., 1994, 59, 5905-5911.

23 For related couplings, see G. A. DeBoos, J. J. Fullbrook, W. M. Owton, J. M. Percy and A. C. Thomas, Synlett, 2000, 963966; G. A. DeBoos, J. J. Fullbrook and J. M. Percy, Org. Lett., 2001, 3, 2859-2861; L. R. Cox, G. A. DeBoos, J. J. Fullbrook, J. M. Percy, N. S. Spencer and M. Tolley, Org. Lett., 2003, 5, 337-339.

24 Fluoroalkenylzinc reagents have an extensive literature: see : D. J. Burton, Z. Yang and P. A. Morken, Tetrahedron, 1994, 50, 29933063. Reference 21 describes one reaction of a reagent related closely to $\mathbf{1 0 .}$

25 R. L. Funk and K. J. Yost, J. Org. Chem., 1996, 61, 2598-2599 describes stannic chloride catalysed cycloadditions of an aldehyde related closely to $\mathbf{1 6}$, though furan was not reported as a successful diene.

26 (a) For a preliminary survey of these species, see: J. Tamariz and P. Vogel, Helv. Chim. Acta, 1981, 64, 188-197. For a more extensive collection of data describing the effects of various Lewis acids, see: O. G. Dealba, J. Chanona, F. Delgado, G. Zepeda, F. Labarrios, R. W. Bates, S. Bott, E. Juaristi and J. Tamariz, Anal. Quim., 1996, 92, 108-117. More recent work applies PES and ab initio, methods: R. Herrera, H. A. Jiménez-Vázquez, A. Modelli, D. Jones, B. Söderberg and J. Tamariz, Eur. J. Org. Chem., 2001, 4657-4699.

27 A. T. Sneden, Synlett, 1993, 313-322.

28 P. Kernen and P. Vogel, Helv. Chim. Acta, 1993, 76, 2338-2343.

29 H. G. Viehe, Z. Janousek and R. Merényi, Acc. Chem. Res., 1985, 18, $148-154$.

30 G. P. Moss, O. C. Keat and G. V. Bondar, Tetrahedron Lett., 1996, 37, 2877-2880.

31 W. Francke and W. Reith, Tetrahedron Lett., 1981, 22, 20292032.

32 S. Y. Wang and W. T. Borden, J. Am. Chem. Soc., 1989, 111, 72827283; S. J. Getty and W. T. Borden, J. Am. Chem. Soc., 1991, 113, 43344335 . Borden shows that the low reactivity of tetrafluoroethylene in $[4+2]$ cycloaddition reactions arises from the facility with which it undergoes $[2+2]$ cycloadditions via biradical pathways. Alkene ground state destabilisation and biradical stabilisation contribute to the effect.

33 Reference 20b reviews related Brønsted and Lewis acid-mediated ring openings of the oxabicyclo[2.2.1] heptenyl template.

34 M. Lautens and W. Klute, Angew. Chem., Int. Ed. Engl., 1996, 35, 442-445.

35 S. Oishi, T. Kamano, A. Niida, Y. Odagaki, H. Tamamura, A. Otaka, N. Hamanaka and N. Fujii, Org. Lett., 2002, 4, 10511054; A. Otaka, H. Watanabe, A. Yukimasa, S. Oishi, H. Tamamura and N. Fujii, Tetrahedron Lett., 2001, 42, 5443-5446.

36 Many of the literature examples take place upon symmetrical systems so electronic influences upon the hydrostannylation reaction have not been defined. For an attempt to detect such influences upon carbopalladtion reactions of the oxabicyclo[2.2.1] heptenyl template, see : C. Montalbetti, M. Savignac, J.-P. Gênet, J.-M. Roulet and P. Vogel, Tetrahedron Lett., 1996, 37, 2225-2228.

37 Duhamel and J-C. Plaquevent, J. Organomet. Chem., 1993, 448, 1-3. 38 S. Sengupta and V. Snieckus, J. Org. Chem., 1990, 55, 5680-5683.

39 R. J. K. Taylor and G. Casy, in Organocopper Reagents: A Practical Approach, ed.R. J. K Taylor, Oxford University Press, Oxford, 1994, p. 39 . 